\title{
Activity-Dependence of Synaptic Vesicle Dynamics
}

\author{
Luca A. Forte, ${ }^{\star} \oplus^{-M i c h a e l ~ W . ~ G r a m l i c h, ~}{ }^{\star}$ and Vitaly A. Klyachko \\ Department of Cell Biology and Physiology, Department of Biomedical Engineering, Washington University, St. Louis, Missouri 63110
}

The proper function of synapses relies on efficient recycling of synaptic vesicles. The small size of synaptic boutons has hampered efforts to define the dynamical states of vesicles during recycling. Moreover, whether vesicle motion during recycling is regulated by neural activity remains largely unknown. We combined nanoscale-resolution tracking of individual synaptic vesicles in cultured hippocampal neurons from rats of both sexes with advanced motion analyses to demonstrate that the majority of recently endocytosed vesicles undergo sequences of transient dynamical states including epochs of directed, diffusional, and stalled motion. We observed that vesicle motion is modulated in an activity-dependent manner, with dynamical changes apparent in $\sim 20 \%$ of observed boutons. Within this subpopulation of boutons, $35 \%$ of observed vesicles exhibited acceleration and $65 \%$ exhibited deceleration, accompanied by corresponding changes in directed motion. Individual vesicles observed in the remaining $\sim 80 \%$ of boutons did not exhibit apparent dynamical changes in response to stimulation. More quantitative transient motion analyses revealed that the overall reduction of vesicle mobility, and specifically of the directed motion component, is the predominant activity-evoked change across the entire bouton population. Activity-dependent modulation of vesicle mobility may represent an important mechanism controlling vesicle availability and neurotransmitter release.

Key words: activity-dependence; presynaptic function; single-particle tracking; synaptic vesicle; vesicle recycling

\section{Significance Statement}

Mechanisms governing synaptic vesicle dynamics during recycling remain poorly understood. Using nanoscale resolution tracking of individual synaptic vesicles in hippocampal synapses and advanced motion analysis tools we demonstrate that synaptic vesicles undergo complex sets of dynamical states that include epochs of directed, diffusive, and stalled motion. Most importantly, our analyses revealed that vesicle motion is modulated in an activity-dependent manner apparent as the reduction in overall vesicle mobility in response to stimulation. These results define the vesicle dynamical states during recycling and reveal their activity-dependent modulation. Our study thus provides fundamental new insights into the principles governing synaptic function.

\section{Introduction}

The majority of central synapses contain a very small pool of releasable vesicles and orchestrate a precisely controlled vesicle recycling program to sustain and modulate release (Harata et al., 2001; Fernández-Alfonso and Ryan, 2006). Vesicle mobility during recycling has received extensive attention because it is thought to represent a rate-limiting step in the recycling process

\footnotetext{
Received Feb. 9, 2017; revised Aug. 8, 2017; accepted Aug. 15, 2017.

Author contributions: L.A.F., M.W.G., and V.A.K. designed research; M.W.G. performed research; L.A.F. and M.W.G. contributed unpublished reagents/analytic tools; L.A.F., M.W.G., and V.A.K. analyzed data; L.A.F., M.W.G., and V.A.K. wrote the paper.

This work was supported in part by Grants from Whitehall foundation, NINDS R01NS089449, R01NS081972, and startup funds from Washington University to V.A.K. We thank Matthiew Strulson for performing pilot experiments for this study.

*L.A.F. and M.W.G. contributed equally to this work.

The authors declare no competing financial interests.

Correspondence should be addressed to Dr. Vitaly A. Klyachko, Department of Cell Biology and Physiology, Department of Biomedical Engineering, 425 South Euclide Avenue, Campus Box 8228, Washington University, St. Louis, M0 63110. E-mail: klyachko@wustl.edu.

DOI:10.1523/JNEUROSCI.0383-17.2017

Copyright $\odot 2017$ the authors $\quad 0270-6474 / 17 / 3710597-14 \$ 15.00 / 0$
}

(Shtrahman et al., 2005; Yeung et al., 2007). The increased demand for vesicles during periods of high-frequency firing has led to the hypothesis that some of the steps in the recycling process are facilitated by neural activity to promote vesicle availability for release. Despite extensive research, however, it remains largely unknown if or how neural activity regulates vesicle mobility and recycling (Maschi and Klyachko, 2015).

Until recently, defining vesicle translocation mechanisms has been limited by the inability of existing techniques to resolve motion of individual vesicles. Early studies performed at room temperature and using predominately bulk assays of vesicle dynamics have led to the conclusion that synaptic vesicles are largely immobile within the terminals or have restricted random dynamics (Henkel et al., 1996; Kraszewski et al., 1996; Lemke and Klingauf, 2005; Gaffield et al., 2006). However, this perceived lack of vesicle mobility has been difficult to reconcile with the findings that newly endocytosed vesicles are often located hundreds of nanometers away from the active zone (AZ; Schikorski and Stevens, 2001; Schikorski, 2014), and that sites of exocytosis and endocytosis are spatially separated (Watanabe et al., 2013, 2014), 
suggesting the need for long-distance vesicle translocation from the endocytic sites to the AZ. Recent findings of large heterogeneity in vesicle properties (Sara et al., 2005; Fredj and Burrone, 2009; Chung et al., 2010; Hua et al., 2011; Raingo et al., 2012; Bal et al., 2013) have underscored the limitations of using bulk measurements to study vesicle dynamics. Indeed, single-vesicle studies from our laboratory (Peng et al., 2012) and from others (Westphal et al., 2008; Kamin et al., 2010; Lee et al., 2012; Park et al., 2012) showed that a majority of vesicles undergo large-scale motion within synaptic terminals over several hundred nanometers, but this extensive vesicle mobility is predominately apparent at physiological temperatures (Gaffield and Betz, 2007; Peng et al., 2012).

Regulation of vesicle mobility and recycling by activity and calcium $\left(\mathrm{Ca}^{2+}\right)$ levels has long been hypothesized, but still remains debated. Single-vesicle synaptopHluorin-based studies have provided strong evidence that synaptic vesicle retrieval is $\mathrm{Ca}^{2+}$-dependent (Leitz and Kavalali, 2011), although initial studies of single-vesicle dynamics did not find any activity-/ $\mathrm{Ca}^{2+}$-dependent changes in vesicle mobility within the terminals at room temperature (Lemke and Klingauf, 2005; Westphal et al., 2008). Yet LTP induction was found to cause a large increase in the mobile fraction of vesicles (Lee et al., 2012), and an increase in vesicle mobility within the terminals was observed upon prolonged $\mathrm{KCl}$ stimulation (Joensuu et al., 2016), suggesting that vesicle dynamics is activity-dependent.

Here we combined single-vesicle tracking with advanced motion analysis tools to characterize the dynamical states of the vesicles during their life cycle. We found that vesicles exhibit complex and heterogeneous dynamics represented by sequences of transient dynamical states. Most importantly, using three complementary motion analysis approaches, we demonstrate that vesicle behavior is activity-dependent, with heterogeneous changes in vesicle motion in subsets of vesicle population. Our results define fundamental properties of vesicle dynamics during recycling and reveal their activity-dependent modulation.

\section{Materials and Methods}

\section{Neuronal cell culture}

Dissociated primary cultures of rat hippocampal neurons from pups of both sexes were created as previously described (Peng et al., 2012). Briefly, neurons from the hippocampus of E19 rat pups were plated at $10,000-20,000$ cells per plate and kept at $37^{\circ} \mathrm{C}$ in Neurobasal media supplemented with B-27 (Invitrogen). For data with stimulus we used 64 sample plates obtained from 12 separate litters with an average of 9 vesicles per sample. For data without stimulus we used 40 sample plates obtained from 8 litters with an average of 8 vesicles per sample. All sample plates were imaged between 12 and $15 \mathrm{~d}$ in vitro. All animal procedures conformed to the guidelines approved by the Washington University Animal Studies Committee.

\section{Fluorescence microscopy}

All experiments were conducted at $37^{\circ} \mathrm{C}$ within a whole-microscope incubator (InVivo Scientific). Fluorescence was excited with a xenon lamp via a $100 \times, 1.4$ NA oil-immersion objective (Olympus), and captured using cooled EM CCD camera (Hamamatsu). Focal plane was continuously monitored, and focal drift was automatically adjusted with $10 \mathrm{~nm}$ accuracy by an automated feedback focus control system (Ludl Electronics). Field stimulation was performed by using a pair of platinum electrodes and controlled by the software via Master- 8 stimulus generator (AMPI). A bath solution contained $1.25 \mathrm{~mm} \mathrm{NaCl}, 2.5 \mathrm{~mm} \mathrm{KCl}, 10 \mathrm{~mm}$ HEPES, $10 \mu \mathrm{M}$ CNQX, $15 \mathrm{~mm}$ glucose with $\mathrm{pH}$ balanced to 7.25 (all from Sigma-Aldrich) and was supplemented with $2.0 \mathrm{mM} \mathrm{CaCl}_{2}, 1.0 \mathrm{~mm}$ $\mathrm{MgCl}_{2}$ for the dye loading and imaging and $0.2 \mathrm{mM} \mathrm{CaCl}_{2}, 2.0 \mathrm{mM} \mathrm{MgCl}_{2}$ to wash excess dye from the sample. SGC5 (10 $\mu$; Biotium) was also added to the bath solution for the dye loading step. Sparse vesicle labeling and functional synapse localization were performed following our previously developed procedures (Peng et al., 2012). First, sparse vesicle labeling was achieved via compensatory endocytosis using a pair of stimuli at $100 \mathrm{~ms}$, followed by a $30 \mathrm{~s}$ incubation for dye loading; the dye solution was washed away for $6 \mathrm{~min}$ using the $0.2 \mathrm{~mm}\left[\mathrm{Ca}^{2+}\right]_{0}$ bath solution; a $2 \mathrm{~mm}\left[\mathrm{Ca}^{2+}\right]_{0}$ solution was then flowed into the sample chamber for $1 \mathrm{~min}$; and samples were then imaged at an exposure rate of $80 \mathrm{~ms}$ (with a total frame rate of $10 \mathrm{~Hz}$ ). We estimated the release probability in our cultures to be $\sim 0.07$ under our experimental conditions (data not shown); therefore a pair of stimuli is likely to label a single or at most two vesicles per synaptic bouton (Peng et al., 2012) in the majority of boutons. Only boutons with a single labeled vesicle were included in all analyses. In the second imaging step, locations of functional synapses were determined using a bout of strong stimulation, 200 stimuli at $30 \mathrm{~Hz}$, in the presence of the dye (Peng et al., 2012) to load the entire releasable pool in all functional synapses and then using another bout of stimulation for $20 \mathrm{~s}$ at $20 \mathrm{~Hz}$ to unload the dye. Only synaptic locations that both uptook and released the dye upon bouts of strong stimulation were considered to represent location of functional synapses.

\section{Image and data analysis}

Detection (subpixel localization) and tracking were performed using a uTrack software package that was kindly provided by Dr. Danuzer's laboratory (Jaqaman, 2008). The input parameters for the PSF were determined by using stationary green fluorescent $40 \mathrm{~nm}$ beads. Localization of functional synapses was performed using ImageJ. Vesicle trajectories spanning $>1 \mu \mathrm{m}$ were excluded from analysis to avoid potential contributions from axonally transported vesicles. Only complete trajectories with vesicle detected in every frame (no gaps) were analyzed (144 tracks of 484 total). Approximately $12-15 \%$ of vesicles disappeared during the observation period for several reasons, which may include exocytosis, endosomal fusion, going out of focus, loss of tracking, etc. Such vesicles, whose tracks were $<20 \mathrm{~s}$ in duration, were not included in our analysis.

\section{Pharmacology}

Okadaic Acid (OA; $100 \mathrm{~nm})$, FK506 (20 $\mu \mathrm{M})$, or Roscovitine $(100 \mu \mathrm{M})$ were applied with a 10 min preincubation of cultures in bath solution supplemented with the given agent.

\section{Statistical analysis}

Statistical analyses were performed in MATLAB. Statistical significance was determined using two-sample two-tailed $t$ test or KolmogorovSmirnov (KS) test, and listed in results section and figure captions where appropriate.

\section{Computational approaches for transient motion analysis of vesicle trajectories}

Bayesian approach to MSD-based analysis of vesicle trajectories (Bayesian analysis)

For a number of reasons [noise, transitions between different dynamical states, decreasing accuracy in the values of the mean square displacement (MSD) with increasing time lag, etc.], a basic analysis of MSD curves is not adequate to robustly detect various dynamical states in singleparticle tracking experiments, thus additional approaches are needed to identify the underlying dynamical states in particle trajectories (Manzo and Garcia-Parajo, 2015). We implemented the approach described previously (Monnier et al., 2012), in which the MSD curve was evaluated for each track; then nonlinear regression of these curves plus Bayesian model selection was used to identify the most likely dynamical states. MSD curves were calculated as the sum of the square of distance between two data points at $[r(i+\tau)-r(i)]^{2}$ for all data points $(i)$, where $\tau$ is the time distance between points called the lag-time, and has been normalized by the number of points for each $\tau$ (Monnier et al., 2012):

$$
M S D(\tau)=\frac{1}{N-\tau} \sum_{i=1}^{N-\tau}[r(i+\tau)-r(i)]^{2} .
$$


Bayesian model selection permits robust multiple hypotheses testing, i.e., it allows to test for more than two hypotheses at the same time. In our case we tested for three different models: pure diffusion, directed motion, and stalled motion. The algorithm returns the percentage of each dynamical state in each track in terms of the time spent in each state. One advantage of this approach is that it does not have any free parameters. A drawback of this analysis is that it does not provide temporal information, i.e., it is not possible to identify which dynamical state occurs along the trajectory at a given time.

\section{Rolling window analysis}

To overcome the drawback of the Bayesian analysis in lacking temporal information, we have used a complementary approach based on a rolling window sliding through the entire trajectory as previously described (Huet et al., 2006). Within the rolling window, it is possible to use different, independent mathematical tools to classify the corresponding portion of the trajectory. One tool we used is the asymmetry parameter derived from the gyration tensor, which quantifies the amount of directed motion and is defined by:

$$
R_{g}(i, j)=\left\langle x_{i} x_{j}\right\rangle-\left\langle x_{i}\right\rangle\left\langle x_{j}\right\rangle .
$$

Where $x_{i}^{\prime}$ s are the Cartesian coordinates and the average values are defined over all $n$ steps of the analyzed trajectory, i.e., $\left\langle x_{i}\right\rangle=\left(\frac{1}{n}\right) \sum_{k=1}^{n} x_{i, k}$. The asymmetry parameter (dimensionless) is then defined in $2 \mathrm{D}$ by:

$$
\text { Asym }=-\log \left(1-\frac{\left(R_{1}^{2}-R_{2}^{2}\right)^{2}}{2\left(R_{1}^{2}+R_{2}^{2}\right)^{2}}\right),
$$

where the gyration radii $R_{1}$ and $R_{2}$ are the square roots of the eigenvalues of the gyration tensor. Higher transient Asym values may reveal periods of directed motion.

In practice when the asymmetry parameter is above a certain threshold we can assign the underlying dynamical state as directed motion. We determine this threshold empirically from our data (not shown). We then distinguish the remaining components of the trajectory as either diffusive or stalled by evaluating the instantaneous diffusion coefficient $D_{\text {inst }}$ according to the guidelines by Huet et al. (2006). The value of $D_{\text {inst }}$ was calculated along a rolling window; specifically a regression line was fitted through the first five points of the corresponding MSD curve and a value for $D_{\text {inst }}$ was obtained. Periods for which $D_{\text {inst }}$ was below a minimum value $D_{\min }$ were classified as stalled periods. Based on our control bead measurements (data not shown), we set the threshold for the stalled motion as follows: $D_{\min }=D_{\text {stalled }}=2 \times 10^{-4} \mu \mathrm{m}^{2} / \mathrm{s}$.

The remaining part of the trajectory was classified as pure diffusion. We distinguished between two components of diffusion, fast and slow ones. This additional sub division of the diffusion state is supported by the distribution of the values of the instantaneous diffusion coefficient (data not shown). We defined a slow component of diffusion when $D_{\text {stalled }}<D_{\text {inst }}<D_{\text {slow }}=0.7 \times 10^{-3} \mu \mathrm{m}^{2} / \mathrm{s}$ and a fast component when $D_{\text {inst }}>D_{\text {slow. }}$. The percentage for each dynamical state derived by this analysis refers to the time spent in each state.

The rolling window analysis is complementary to the Bayesian analysis in two aspects. The first one is that the global identified dynamical states are very similar between the two approaches (see Fig. 2), giving robustness to the overall result. The second is that with the rolling window analysis it is possible to extract the temporal information (i.e., the temporal sequence of the states), allowing to color-code each trajectory according to the underlying dynamical states (see Figs. 1-3).

Spatial analysis of vesicle motion based on van Hove correlation function

The van Hove correlation function (VHCF) is a widely used tool in diffusion theory that examines the spatial aspects of particle motion (Del Pópolo and Voth, 2004; Ahmed and Saif, 2014; Skaug et al., 2014).

The VHCF represents the probability distribution of displacements $(x$ or $y$ coordinates in 2D) at different time lags:

$$
P(\Delta x, \tau) \text {, where } \Delta x(\tau)=\Delta x(t+\tau)-\Delta x(t) .
$$

The theory predicts a Gaussian distribution for particles undergoing pure diffusion (thermal Brownian motion), although the width of the Gaussian distribution may increase with $\tau$. The histogram of $\Delta x(\tau)$ can show deviations from Gaussianity, specifically exhibiting longer tails. Those deviations can be ascribed either to periods of directed motion since particles occasionally take longer athermal jumps due to active transport or to a heterogeneous environment. To quantify the Gaussianity of the VHCF, we used the kurtosis, which is the fourth moment of a probability distribution. Our normalization was such that the kurtosis is equal to three for a theoretical Gaussian distribution and always bigger than three for any non-Gaussian distribution. For real data, absolute values of kurtosis are not easily comparable between different datasets because of noise and sensitivity to outliers; instead the values of the kurtosis are typically compared within the same dataset, i.e., before and during stimulation. In addition, the SD of the VHCF represents a measure of vesicle displacement across different time lags.

\section{Transient motion correlation analysis}

Quantification of frame-to-frame displacement. We used a three-frame moving average, which reduces noise but does not strongly affect temporal resolution. Specifically we use a forward biased moving average, as opposed to a symmetric moving average:

$$
<x_{i}>=\frac{1}{3} \sum_{j=i}^{i+3} x_{j} ;<y_{i}>=\frac{1}{3} \sum_{j=i}^{i+3} y_{j} .
$$

Raw track data can often have gaps within a single track. Gaps are typically caused by the inability of the detection algorithm to fit the vesicle image to the PSF within the required precision levels. This could arise from multiple factors, including noise or broadening of the vesicle image due to going out of focus. We fill such gaps of one or two missing frames, using a moving average analysis window. The position for the missing frame $(x(t), y(t))$ is determined as the mean of four frames ( 2 before and 2 after the missing frame). Tracks with gaps $>2$ frames were not considered further.

Two metrics are used to quantify changes in vesicle displacement: velocity $(v)$ and direction of displacement, which we refer to as angular displacement $(\theta)$. Velocity is calculated as the distance between the vesicle position at a previous time-point and the current vesicle position, divided by the frame rate $(100 \mathrm{~ms})$ :

$$
v_{i}=\frac{1}{100} \sqrt{\left(x_{i-1}-x_{i}\right)^{2}+\left(y_{i-1}-y_{i}\right)^{2}} .
$$

The direction of the displacement $(\theta)$ is determined symmetrically as the difference between the direction of the previous displacement and the direction of the next displacement:

$$
\begin{aligned}
\theta & =a \cos \left[\frac{\Delta x_{-i} \Delta x_{+i}+\Delta y_{-i} \Delta y_{+i}}{R_{-i} \cdot R_{+i}}\right] \\
\Delta x_{-i} & =x_{i}-x_{i-1} ; \Delta y_{-i}=y_{i}-y_{i-1}, \\
\Delta x_{+i} & =x_{i+1}-x_{i} ; \Delta y_{+i}=y_{i+1}-y_{i} \\
R_{+/-i} & =\sqrt{\Delta x_{+1-i}^{2}+\Delta y_{+1-i}^{2}}
\end{aligned}
$$

we note here that this method only considers the absolute value of the angular displacement and ignores sign value $( \pm)$. We chose this method because in our analysis we are interested in deviations from directionality $(\theta=0)$ and not any specific orientation change.

\section{Determination of different types of motion}

We use a correlation analysis to determine when vesicles are engaging in different types of motion (Gramlich and Klyachko, 2017). We define three types of motion: fast directed motility, intermediate motility, and pausing. Each type of motion is based on instantaneous vesicle velocity $(v)$ and the angular displacement between frames $(\theta)$. The thresholds for defining directed motion are based on the known motility parameters of myosin- $\mathrm{V}$, the main synaptic vesicle-associated member of myosin family found in presynaptic terminals of central neurons. Individual 
myosin-V motors have an instantaneous velocity of at least $0.20 \mu \mathrm{m} / \mathrm{s}$ in actin gliding assays in vitro (Krementsov et al., 2004). Individual motors also walk strait along actin filaments with minimal angular displacement, and any significant angular displacement $\left(\theta>80^{\circ}\right)$ would thus not likely be due to myosin- $\mathrm{V}$-dependent transport along an actin filament. Finally, the minimum number of frames during which the motion have to be observed to qualify for a directed motion is based on approximating the minimum expected distance per run from the known run-length of Myosin-V (Krementsov et al., 2004). Consequently, we set the rules for each type of motion based on these molecular mechanical properties of myosin-V:

A fast directed motility is defined by satisfying all of following rules:

(1) Instantaneous velocity: $v>0.2 \mu \mathrm{m} / \mathrm{s}$ is required for every frame.

(2) Instantaneous angular displacement: $\theta<80^{\circ}$ is required for every frame.

(3) Duration: a minimum of 4 frames.

A pause is defined by satisfying all of the following rules:

(1) Instantaneous velocity and angular displacement: If $\theta<80^{\circ}, v<$ $0.20 \mu \mathrm{m} / \mathrm{s}$. If $\theta>80^{\circ}, v<0.25 \mu \mathrm{m} / \mathrm{s}$. If $\theta=0^{\circ}, v<0.12 \mu \mathrm{m} / \mathrm{s}$.

(2) Average angular displacement: $\langle\theta\rangle\rangle 70^{\circ}$. We note that a completely random motion have an average change in angle of $60^{\circ}$; we chose a slightly more stringent requirement because of the limited number of frames per pause that would make the average less accurate.

(3) Duration: a minimum of 4 frames.

An intermediate motion represents all the remaining motion.

The resulting classification accounts for all track data without overlap (Gramlich and Klyachko, 2017, their Fig. S1, see example track). Further, this algorithm accounts for the reduction in velocity and angular displacement caused by the 3 -frame moving average by setting lower thresholds for each limit (Gramlich and Klyachko, 2017).

Spatial displacement analysis using 95\% radius

Spatial displacement analysis was performed by calculating the mean position of all data within a given time-range $(0-10 \mathrm{~s}$ or $10-20 \mathrm{~s})$, and then by calculating the radius $(R)$ from the mean position so that $95 \%$ of all track points are $<R$. The displacement was then evaluated in two ways: Figure $1 I$ reports an ensemble average of all tracks. Figure $4 A$ determines the cumulative distribution of radii for all tracks that is fit using an exponential recovery $\left(1-\exp ^{-x / r}\right)$. The resulting mean value $(r)$ then represents the expected probable radius for any individual vesicle track (Fig. $4 B$ ).

\section{Results}

Detection and tracking of individual synaptic vesicles in small central synapses

To reliably detect and track single fluorescently-labeled synaptic vesicles, we used a nanoscale resolution imaging approach we recently developed (Peng et al., 2012), which incorporates established single-particle tracking tools (Jaqaman, 2008). Synaptic vesicles were loaded with a fluorescent lipophilic dye SGC5 (Wu et al., 2009) via compensatory endocytosis using a pair of stimuli at $100 \mathrm{~ms}$ to ensure sparse vesicle labeling. Due to low release probability of hippocampal synapses at $37^{\circ} \mathrm{C}(p \sim 0.07$, data not shown), this sparse labeling protocol results in staining of only a single vesicle in the majority of boutons (Peng et al., 2012); to avoid ambiguity in vesicle tracking caused by overlapping vesicle trajectories, we excluded from analysis a small subset of boutons in which more than one vesicle was labeled. Because only a single vesicle is labeled per bouton, the observed population of vesicles represents distribution of vesicle properties across population of boutons. To make sure that only functional boutons were analyzed, acquisition of each single-vesicle movie was followed by two control measurements with strong staining/destaining procedures (Peng et al., 2012); only functional boutons that could load and release the dye were analyzed further. With this approach, individual synaptic vesicles were reliably tracked with $\sim 20 \mathrm{~nm}$ localization precision in hippocampal terminals at $37^{\circ} \mathrm{C}$. We observed that a majority of synaptic vesicles underwent largescale motion (spanning $\sim 100-800 \mathrm{~nm}$ ) during recycling at $37^{\circ} \mathrm{C}$ and exhibited complex sequences of dynamical behaviors (Fig. 1A). However, the analysis of the instantaneous diffusion coefficient alone was insufficient to capture the apparent complexity of vesicle dynamics (Fig. 1B). We thus used additional mathematical tools to characterize vesicle dynamical states during recycling.

\section{Characterization of vesicle dynamical states in central synapses}

In our first approach to analyze vesicle dynamical states we apply a motion analysis method based on nonlinear regression of the MSD curves of individual vesicle tracks with Bayesian model selection to classify different types of vesicle behavior. We chose the Bayesian approach because a basic analysis of MSD curves is not adequate to robustly detect various dynamical states in single-particle tracking experiments due to noise, transitions between different dynamical states, decreasing accuracy in the values of the MSD with increasing time lag etc. (Manzo and Garcia-Parajo, 2015; Fig. 1B).

Further, Bayesian model selection permits multiple hypotheses testing, which allows us to define the number of available states. We followed a previously defined approach for analyses of vesicle transport (Monnier et al., 2012), in which the MSD curve was evaluated for each track; then nonlinear regression of these curves plus Bayesian model selection was used to identify the most likely dynamical states. This approach permits robust detection of stalled, diffusive and directed motion epochs within individual vesicle trajectories (Fig. $1 A-C$; see Materials and Methods).

First, to establish the vesicle dynamical states in the absence of stimulation, we applied this analysis to individual vesicle trajectories in two $10 \mathrm{~s}$ intervals $(0-10 \mathrm{~s}$ and $10-20 \mathrm{~s})$ under resting conditions and determined the duration of each dynamical state averaged across the vesicle population. We observed that vesicles spent on average $13 \pm 7 \%$ of the time in directed motion, $52 \pm$ $13 \%$ in diffusive motion and $35 \pm 13 \%$ in stalled motion (Fig. $1 C$; we note that only complete tracks with no gaps could be used in this analysis, $N=37$ ). In resting terminals, this mixture of vesicle behaviors remains largely unaltered for the two subsequent $10 \mathrm{~s}$ periods of observation, reflecting the stability of vesicle dynamics over this time period (Fig. 1C).

Although this Bayesian analysis provides a robust classification of vesicle motion without free parameters, a drawback of this analysis is that it does not identify when dynamical state occurs along the trajectory at a given time. We thus confirmed these results using an independent but complementary motion analysis approach based on a rolling window method to classify different types of motion and their corresponding portion of the trajectory (Fig. 1 D, E; Materials and Methods; Huet et al., 2006).

We used the asymmetry parameter, derived from the gyration tensor, to quantify the amount of directed motion (see Materials and Methods). To classify the remaining parts of the trajectory, we evaluated the instantaneous diffusion coefficient that permits reliable quantification of stalled and diffusive types of vesicle motion. We observed a very close agreement in distribution of vesicle dynamical states between the Bayesian and rolling window analysis approaches (Figs. 2, compare $D, F$ and $G, H$ ). The rolling window approach further allowed us to distinguish between fast and slow components of diffusion (Fig. $1 F$ ). As with Bayesian analysis, we did not observe any statistical differences between the first and second $10 \mathrm{~s}$ periods of the trajectories in the distribution 
A

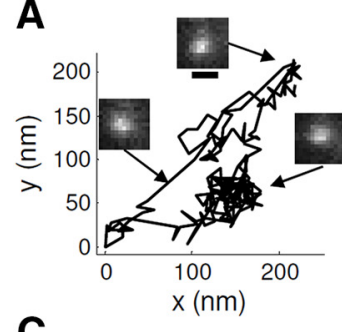

B

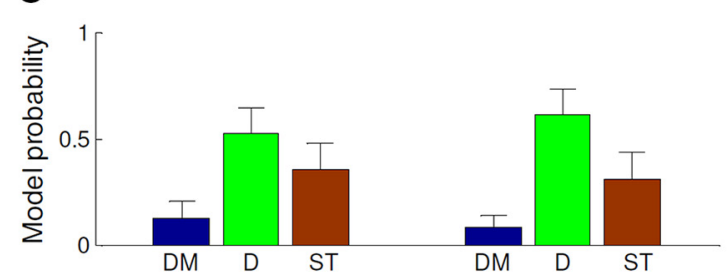

G

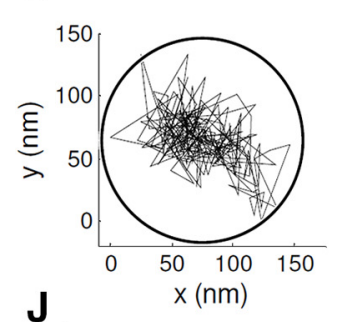

H
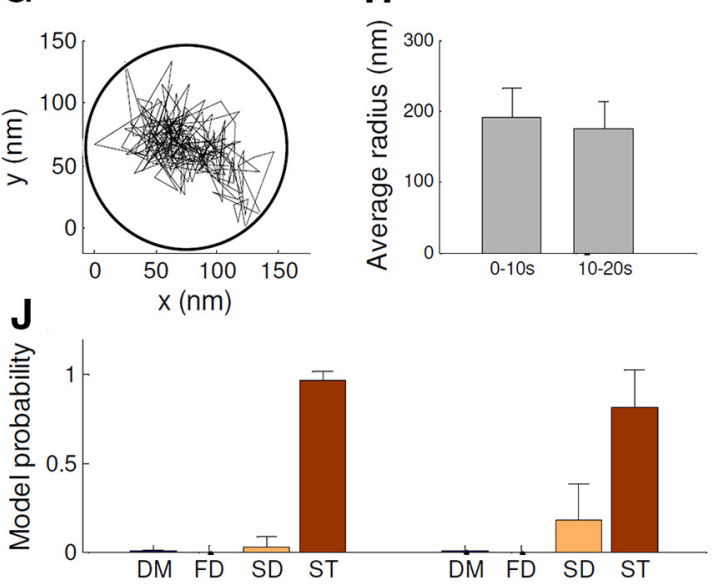

D
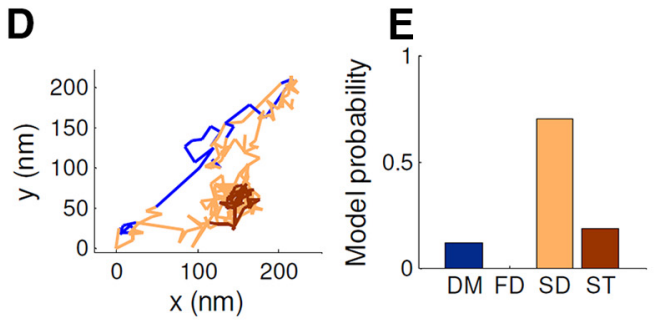

F

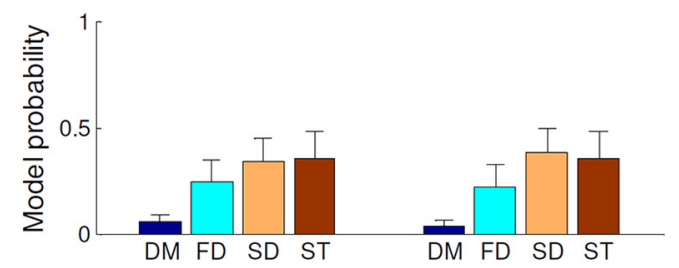

I

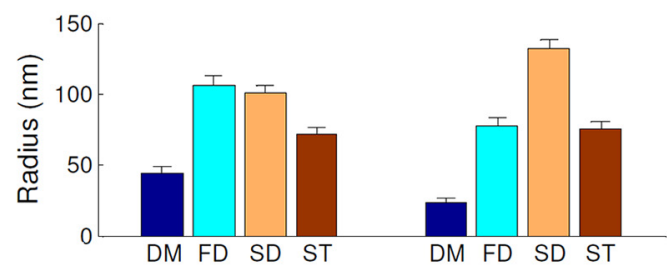

K

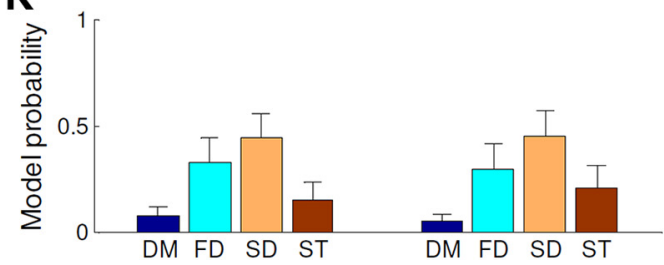

Figure 1. Characterization of vesicle dynamical states in the absence of stimulation. $A$, Example of a synaptic vesicle trajectory moving within a synapse during a $20 \mathrm{~s}$ observation with images of the vesicle at different time points along the trajectory corresponding to different dynamical states. Scale bar, $300 \mathrm{~nm}$. B, Instantaneous diffusion coefficient (blue) and MSD (green) curves for the trajectory in $A$. C, Probability of occurrence of various dynamical states in the absence of stimulation within the vesicle tracks (in terms of time spent in the given state) as determined using Bayesian analysis. Bar graphs are plotted separately for $0-10 \mathrm{~s}$ (left) and 10-20 s (right) periods of observation and represent directed motion (DM), diffusion (D), and stalled motion (ST). D, The same trajectory as in $\boldsymbol{A}$ color-coded according to the rolling window analysis. Four dynamical states were differentiated with rolling window analysis, which included DM, ST, and D, which was subcategorized into fast diffusion (FD) and a slow diffusion (SD). In this particular trajectory, no FD was detected. $\boldsymbol{E}$, Probability of occurrence of various dynamical states within the trajectory in $\boldsymbol{D}$ as determined using rolling window analysis. $\boldsymbol{F}$, Same as $\boldsymbol{E}$ for all vesicle tracks in the absence of stimulation. Bar graphs in each panel correspond to the $0-10 \mathrm{~s}$ period (left) and $10-20 \mathrm{~s}$ period (right). The analyses were performed track by track and then averaged. Only complete tracks with vesicles detected in every frame could be analyzed ( $N=37$ tracks). $\mathbf{G}-\mathbf{I}$, Spatial analysis of vesicle displacement based on the radius of the circle encompassing $95 \%$ of vesicle trajectory, as described previously (Peng et al., 2012). Sample vesicle track with the circle shown (G). The circle size was evaluated for each track separately for the $0-10$ s and $10-20$ s periods and averaged among all the tracks $(\boldsymbol{H})$. Vesicle displacement (radius of the $95 \%$ circle) was determined for each dynamical state $(\boldsymbol{I})$ as defined by the rolling window analysis in $\boldsymbol{F}$. $\boldsymbol{J}, \boldsymbol{K}$, The subpopulation of mostly stalled vesicles $(>70 \%$ stalled in the $0-10 \mathrm{~s}$ period) exhibit few dynamical states $(\boldsymbol{J})$, whereas the remaining vesicles exhibit a large distribution of dynamical states $(\boldsymbol{K})$, as determined by the rolling window analysis. Error bars represent $95 \% \mathrm{Cl}$.

of dynamical states in the rolling window analysis (Fig. 1F; Directed, $p=0.33$; Fast diffusion, $p=0.71$; Slow diffusion, $p=$ 0.55 ; Stalled, $p=0.97 ; t$ test, degrees of freedom $=36$ ).

Although the above analyses defined the temporal characteristics of vesicle dynamical states, we sought to further define these states using a spatial measure of vesicle displacement. This measure was defined as the radius of a circle that encompasses $95 \%$ of the vesicle trajectory (Fig. $1 G$ ) in a given dynamical state. It thus represents the total distance traveled in each dynamical state by a given vesicle that was than averaged across the vesicle population. The beginning and the end points for each state were determined using the rolling window approach. Similarly to the above analyses, the total spatial displacement was not significantly different in the first and second $10 \mathrm{~s}$ periods of the trajectories under resting (no stimulation) conditions (Fig. $1 H ; p=0.58$; $t$ test, degrees of freedom $=36$ ). As could be expected from the higher velocity of directed motion and fast diffusion, their contributions to spatial extent of vesicle motion were more pronounced compared with the temporal analyses (Fig. 1I). We note however, that because this displacement measure depends on both vesicle velocity and time-spent in a given motion, the resulting radius value for directed motion is smaller than for stalled or diffusive motion because vesicles on average spent much less time in directed motion than in the two other types of motion.

We noted an apparent heterogeneity in mobility of observed vesicles, with a majority being highly mobile (27/37 boutons), but a subset of vesicles remaining largely immobile throughout the observation period in the remaining subpopulation of boutons (10/37 boutons), consistent with a heterogeneity also observed in several previous studies (Westphal et al., 2008; Kamin et al., 2010; Lee et al., 2012; Park et al., 2012). To further investigate the presence of the distinct dynamical subpopulations, we used a rolling window analysis and considered separately the "stalled" vesicles defined as those with $>70 \%$ of stalled motion during the first $10 \mathrm{~s}$ of 

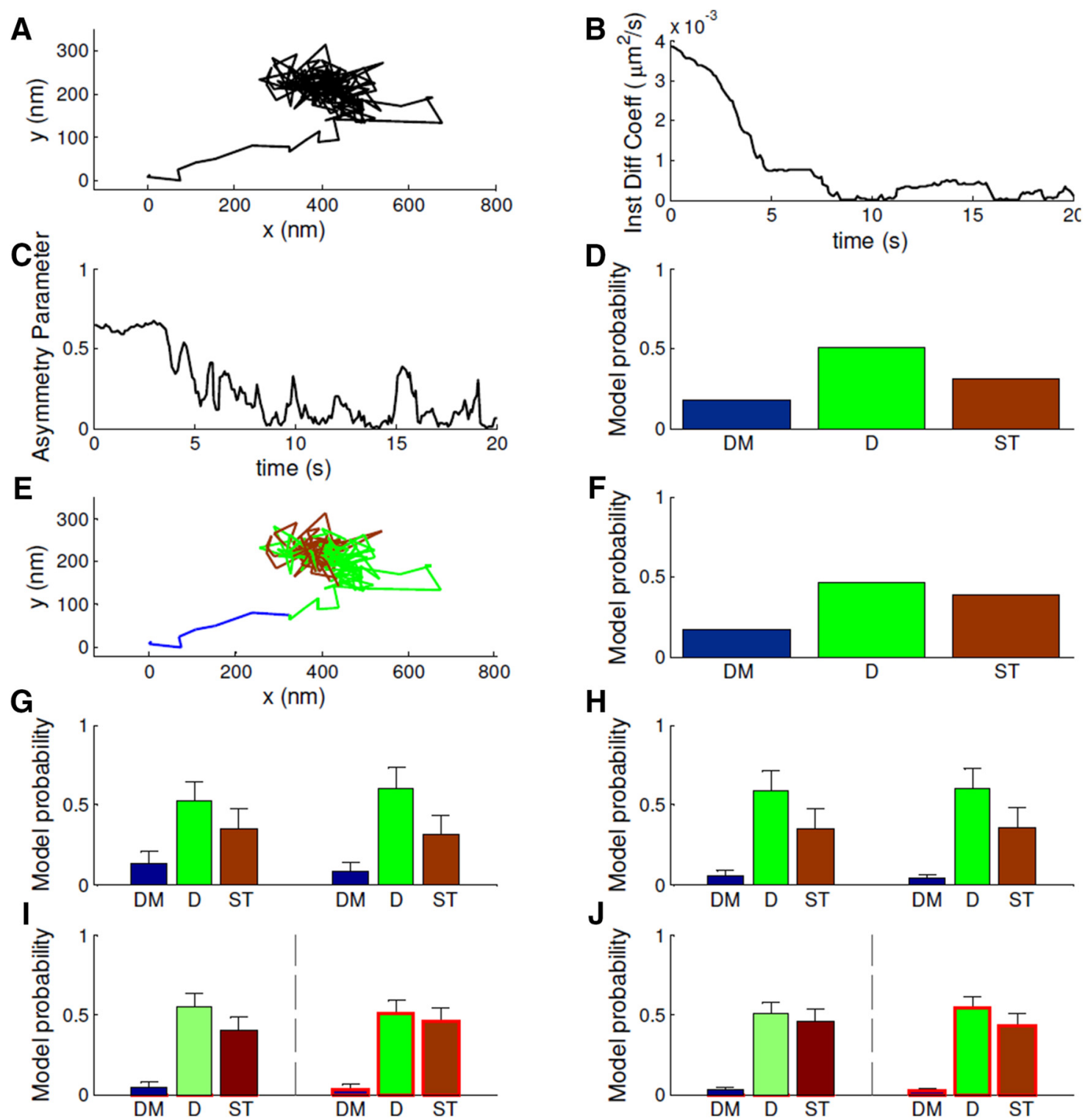

Figure 2. Close agreement between Bayesian and rolling window analyses. $\boldsymbol{A}-\boldsymbol{C}$, Sample vesicle track $(\boldsymbol{A})$ and the corresponding plots of the instantaneous diffusion coefficient $(\boldsymbol{B})$ and the asymmetry parameter $(\boldsymbol{C}) \cdot \boldsymbol{D}-\boldsymbol{F}$, The probabilities of occurrence of each dynamical state for the entire $20 \mathrm{~s}$ duration of the track shown in $\boldsymbol{A}$, as determined by Bayesian analysis $(\boldsymbol{D})$ or rolling window analysis $(\boldsymbol{F})$. The two approaches show a close agreement. The sample track from $A$ is color-coded according to the underlying dynamical mode as determined by the rolling window analysis which provides temporal information. The results of the rolling window analysis are shown without differentiating between fast diffusion and slow diffusion (unlike the main text) with the purpose to demonstrate that both analyses agree well. $\mathbf{G}, \boldsymbol{H}$, The probabilities of occurrence of each dynamical state for the $0-10 \mathrm{~s}$ and $10-20 \mathrm{~s}$ periods averaged across vesicle population in the absence of stimulation, as determined by Bayesian analysis $(\boldsymbol{G})$ or rolling window analysis $(\boldsymbol{H})$. The two approaches show a close agreement. $\boldsymbol{I}, \boldsymbol{J}$, Same as $\boldsymbol{G}$ and $\boldsymbol{H}$ in the presence of stimulation during $10-20$ s period. Error bars represent $95 \% \mathrm{Cl}$.

observation. This stalled vesicle subpopulation comprised nearly a third of all observed vesicles and had a tendency to remain stalled or to develop a small component of slow diffusion over time, but exhibited no apparent fast diffusion or directed motion during the duration of observation (Fig. 1J). In contrast, the remaining vesicles exhibited a full range of dynamical behaviors including directed motion and fast diffusion (Fig. $1 K$ ).

Together these results suggest that recently endocytosed vesicles undergo a complex and heterogeneous set of dynamical behaviors during recycling.

\section{Neuronal activity evokes heterogeneous changes in vesicle dynamics}

Earlier studies of vesicle dynamics performed at room temperature or using bulk measurements (Henkel et al., 1996; Kraszewski et al., 1996; Lemke and Klingauf, 2005; Gaffield et al., 2006) did not observe any measurable changes in vesicle motion evoked by neural activity. Here we examined this question at the level of individual vesicles at $37^{\circ} \mathrm{C}$ by comparing dynamical states of the vesicles for $10 \mathrm{~s}$ before and during high-frequency stimulation (200 stimuli at $20 \mathrm{~Hz}$ ). Initial visual examination of vesicle tracks revealed that high-frequency stimulation at $37^{\circ} \mathrm{C}$ evoked changes in vesicle motion in a subset of boutons ( $\sim 18 \%, 20 / 109$ total; Fig. 3 ), whereas the vesicles in the remaining observed boutons did not exhibit a visually apparent response to stimulation.

A more quantitative examination of the subpopulation of boutons that exhibited an apparent response to stimulation by both Bayesian analysis and the rolling window analysis indicated that response to stimulation was heterogeneous even within this subpopulation: a majority of these vesicles $(\sim 65 \%)$ exhibited a 

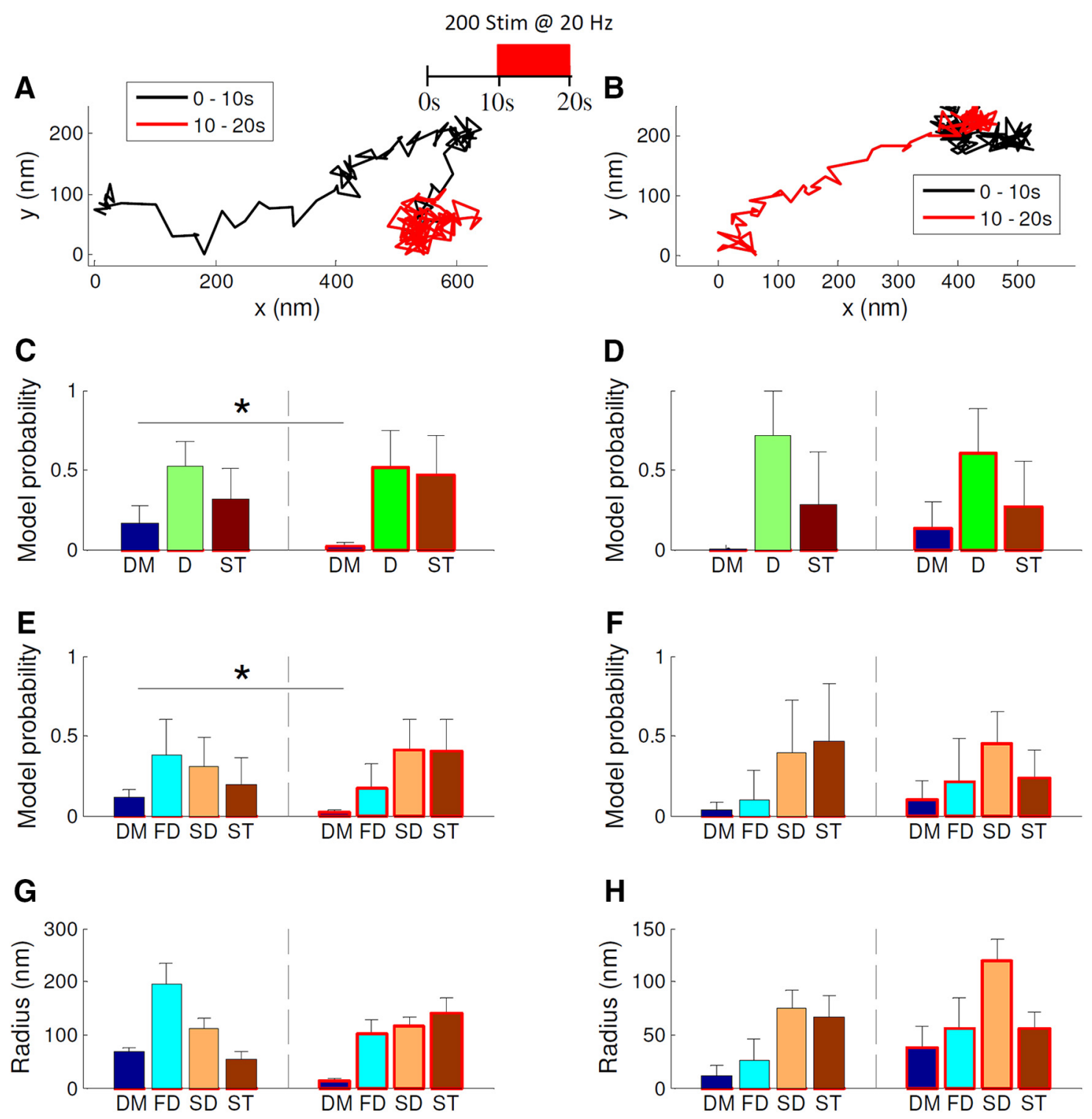

Figure 3. Activity evokes heterogeneous changes in vesicle dynamics. $\boldsymbol{A}, \boldsymbol{B}$, Sample vesicle trajectories exhibiting a visually apparent activity-dependent behavior: deceleration (A) and acceleration (B). Colors of the trajectories' components correspond to the absence of stimulus (black, $0-10 \mathrm{~s}$ ) or presence of the stimulus (red, $10-20 \mathrm{~s}$ ). $\boldsymbol{C}, \boldsymbol{D}$, Probability of occurrence of various dynamical states (in terms of time spent in the given state) based on the Bayesian analysis for the vesicle tracks exhibiting apparent deceleration ( $\boldsymbol{C}$ ) or acceleration ( $\boldsymbol{D})$. Each bar graph shows vesicle dynamical states before stimulation ( $0-10$ s period; left) and during stimulation ( $10-20 \mathrm{~s}$; right). The red edges around the bars indicate the presence of the stimulus. $n=13(\boldsymbol{C})$ and $7(\boldsymbol{D})$ tracks. $\boldsymbol{E}, \boldsymbol{F}$, Same vesicle populations as $\boldsymbol{C}$ and $\boldsymbol{D}$ analyzed with the rolling window analysis. $\mathbf{G}, \boldsymbol{H}$, Same as $\boldsymbol{C}$ and $\boldsymbol{D}$ analyzed using spatial analysis based on $95 \%$ circle. Note that because the spatial displacement values cannot be normalized, the distributions of displacements for different dynamical states cannot be compared statistically. ${ }^{*} p<0.05$, two-tailed $t$ test. Error bars represent $95 \%$ Cl.

"deceleration" with reduction in directed motion and a trend toward increased stalled state (Fig. $3 A, C, E, G$ ), whereas a smaller subset of vesicles $(\sim 35 \%)$ exhibited an apparent "acceleration" of motion with a trend toward an increase in directed motion component (Fig. $3 B, D, F, H$ ). In contrast, we did not observe apparent acceleration or deceleration of motion in vesicle tracks in which no stimulation was applied (data not shown), which strongly supports the notion that vesicle acceleration/deceleration are induced by activity. The opposing changes in vesicle dynamics we observed in response to activity may be averaged out when analyzed across the entire vesicle population, which could explain why such activity-dependent changes have not been observed previously. Indeed, we found that changes in vesicle behavior during stimulation were no longer detectable in the averaged vesicle behavior across the entire population by either type of analysis (Fig. 2I,J). These results suggest that vesicle mobil- ity is modulated by neural activity in a subset of the vesicle population, but heterogeneity of changes in vesicle dynamics makes it difficult to detect in a whole-population analysis, suggesting a need for additional analysis tools to reveal these changes.

\section{Activity-dependent changes in vesicle dynamics detected across entire vesicle population}

Motion analyses we have described in the previous sections primarily focus on a temporal view on vesicle dynamics. To extend the above observations we adopted mathematical tools that examine the spatial behavior of vesicles. First, a global spatial measure (see Materials and Methods, Spatial displacement analysis using 95\% radius) of vesicle mobility applied to the entire vesicle population indicated a significant reduction in total vesicle displacement during stimulation at $37^{\circ} \mathrm{C}$ (Fig. $4 A, B$ ). In line with previous studies (Henkel et al., 1996; Kraszewski et al., 1996; 

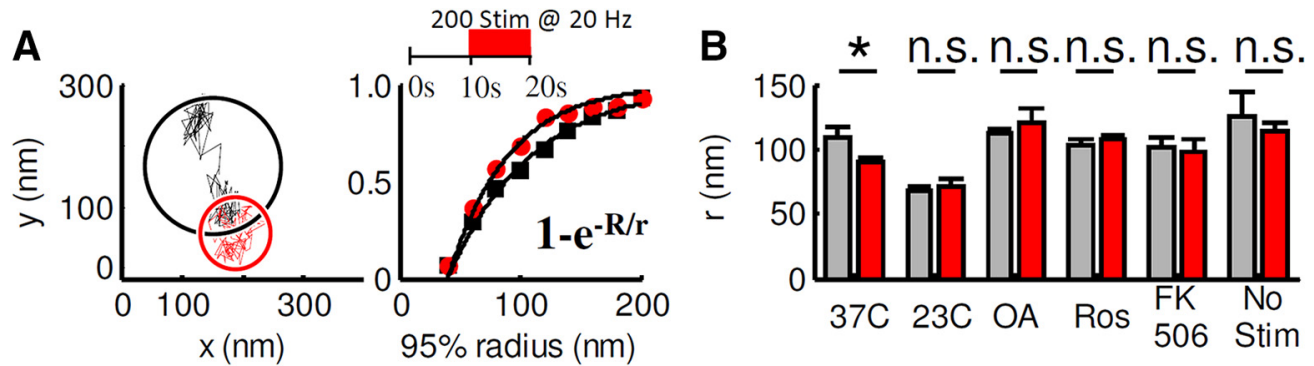

C

D
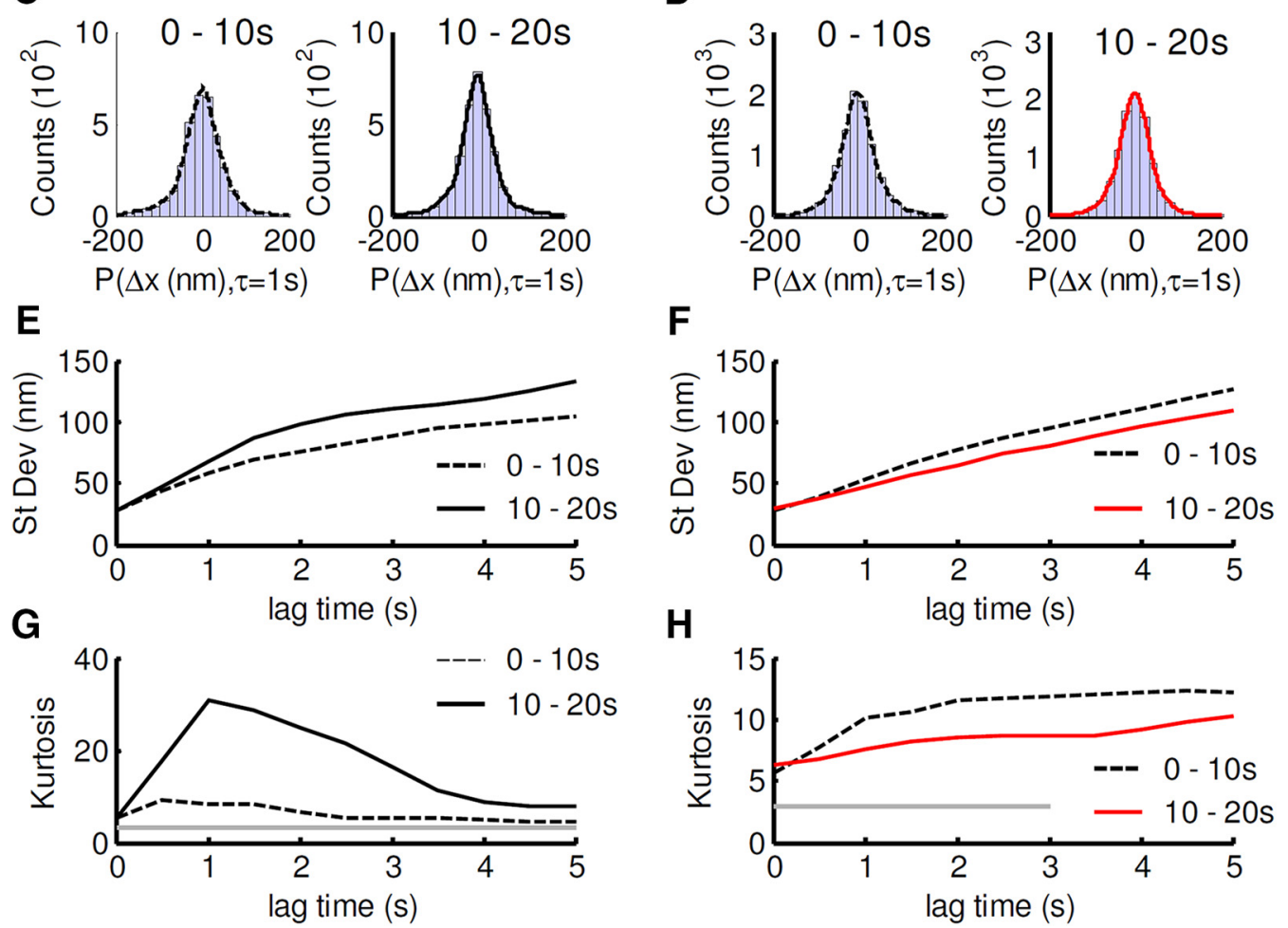

Figure 4. Activity-dependent changes in vesicle dynamics detected across entire vesicle population. $\boldsymbol{A}$, Spatial analysis of changes in vesicle displacement evoked by activity based on the circle encompassing $95 \%$ of vesicle trajectory. Analysis was performed for each track and averaged among all the tracks $(n=54)$ for the $0-10 \mathrm{~s}$ period before stimulation and $10-20 \mathrm{~s}$ period during stimulation. Plotted values were determined from fitting cumulative distributions as described in Materials and Methods, and represent changes in mean circle radius at baseline $(0-10 \mathrm{~s}$; black) or during activity (10-20 s; red). Fits (solid lines) are single exponential recovery. $\boldsymbol{B}$, Changes in spatial vesicle displacement analyzed as in $\boldsymbol{A}$ in different conditions at baseline $0-10$ s ( $g$ ray) and during stimulation $10-20 \mathrm{~s}$ (red) periods. Vesicle displacement was reduced at $23^{\circ} \mathrm{C}$ as compared $37^{\circ} \mathrm{C}$. The effect of activity on vesicle displacement at $37^{\circ} \mathrm{C}$ was lost by lowering the temperature to $23^{\circ} \mathrm{C}$, or in the presence of $0 \mathrm{~A}(100 \mathrm{~nm})$, Roscovitine (Ros; $100 \mu \mathrm{m})$, or FK506 $(20 \mu \mathrm{m}) . N(\mathrm{Stim})=54 ; N\left(23^{\circ} \mathrm{C}\right)=14 ; N(0 \mathrm{~A})=23 ; N($ Ros $)=48 ; N(F K 506)=18 ; N(\mathrm{No}$-stim) $=29$. Note that analysis of cumulative distributions rather than ensemble averages was implemented here to reduce the effects of outliers, leading to different baseline values of displacement compared with Figure $1 \mathrm{H}$. $C, D$, Spatial analysis of changes in vesicle displacement evoked by activity based on the VHCF. Sample histograms of the displacement distributions of the coordinate $x$ at time lag $\tau=1 \mathrm{~s}$ for the first $(0-10 \mathrm{~s})$ and the second $(10-20 \mathrm{~s})$ periods of vesicle trajectories in the absence of stimulation $(\boldsymbol{C})$ or when stimulation was applied during $10-20 \mathrm{~s}$ period (D); superimposed with each histogram are the corresponding probability density functions (estimated via kernel smoothing). The red line indicates the presence of the stimulus. $\boldsymbol{E}-\boldsymbol{H}$, Two metrics to quantify different aspects of the VHCF, the SD $(\boldsymbol{E}, \boldsymbol{F})$ and the kurtosis $(\boldsymbol{G}, \boldsymbol{H})$, plotted for the first $(0-10 \mathrm{~s})$ and second $(10-20 \mathrm{~s})$ periods of all tracks in the absence $(\boldsymbol{E}, \boldsymbol{G})$ and presence $(\boldsymbol{F}, \boldsymbol{H})$ of stimulation during the $(10-20 \mathrm{~s})$ period highlighted by red lines. ${ }^{*} p<0.05$, two-tailed $t$ test. Error bars represent $95 \% \mathrm{Cl}$.

Lemke and Klingauf, 2005; Gaffield et al., 2006), this spatial extent of mobility was significantly reduced by lowering the temperature to $23^{\circ} \mathrm{C}$, and, most importantly, the stimulationdependent changes in mobility were no longer detectable at room temperature (Fig. 4B).

We sought to confirm activity-dependent changes in the spatial extent of vesicle mobility by comparing the total vesicle displacement in several conditions previously suggested to regulate vesicle mobility at different activity levels. In particular, the nonspecific phosphatase inhibitor Okadaic Acid (OA) was suggested to increase basal vesicle mobility in the NMJ (Gaffield et al., 2006), whereas modulation of phosphorylation/dephosphorylation levels of synapsin I by cdk 5 and calcineurin, respectively, was suggested to regulate vesicle mobilization during elevated ac- tivity in central synapses (Chi et al., 2003). We found that changing phosphorylation/dephosphorylation balance with $\mathrm{OA}$, or more specifically by inhibition of cdk 5 with roscovitine or inhibition of calcineurin with FK506, did not significantly affect basal vesicle mobility at $37^{\circ} \mathrm{C}$ (Fig. $4 \mathrm{~B}$ ), but all of these agents eliminated changes in total vesicle displacement evoked by stimulation (Fig. 4B). Finally, we previously reported that actin destabilization with latrunculin-A had a strong effect on directionality and speed of vesicle motion within synaptic boutons both at baseline and during high-frequency stimulation (Gramlich and Klyachko, 2017). Together these results support the above observations of activity-dependence of vesicle mobility and suggest that it is controlled by several different molecular pathways. 
We next turned to a more sensitive motion analysis tool to extend our observations of activity-dependent changes in vesicle mobility at $37^{\circ} \mathrm{C}$. This tool is based on the so-called VHCF, which examines the spatial aspects of vesicle motion (see Materials and Methods; Del Pópolo and Voth, 2004; Ahmed and Saif, 2014; Skaug et al., 2014). The SD of the VHCF represents a measure of vesicle displacement across different time lags. We found that in the absence of stimulation, the SD of the VHCF had a tendency to increase over time and was larger in the second part of the tracks $(10-20 \mathrm{~s})$ compared with the first part $(0-10 \mathrm{~s})$ for all time lags (Fig. 4C,E). In contrast, we observed the opposite changes when stimulation was present, i.e., the SD of the VHCF was reduced during the stimulation period (10-20 s) relative to the preceding no-stimulation period $(0-10 \mathrm{~s}$; Fig. $4 D, F)$. The increase in SD and kurtosis (see below) in the absence of stimulation could be due to photo-bleaching over time, and/or these changes may also reflect a small subset of vesicles that drift toward the axon over time and out of focus, a phenomenon which we and others observed previously (Darcy et al., 2006; Staras et al., 2010; Gramlich and Klyachko, 2017). Further, these results can be interpreted to indicate that the average spread of displacements was reduced during stimulation compared with resting conditions. This observation supports the results of the above spatial analysis based on the $95 \%$ radius (Fig. $4 A, B$ ) that vesicle displacement is reduced during stimulation.

VHCF analysis is also a powerful approach often used to detect deviations of the particle motion from pure passive diffusion (Del Pópolo and Voth, 2004; Ahmed and Saif, 2014; Skaug et al., 2014). The theory predicts a Gaussian distribution of the VHCF for particles undergoing pure diffusion (i.e., thermal Brownian motion). However, if particles move in highly heterogeneous environments or undergo active transport with longer athermal jumps, the histogram of displacements (i.e., the VHCF) shows deviations from Gaussianity. To quantify the Gaussianity of the VHCF, a statistical parameter known as kurtosis is commonly used, which is the fourth moment of a probability distribution (Del Pópolo and Voth, 2004; Ahmed and Saif, 2014; Skaug et al., 2014). The kurtosis values are normalized such that the kurtosis is equal to 3 for a theoretical Gaussian distribution and is $>3$ for any non-Gaussian distribution (see Materials and Methods). Our analysis showed that in the absence of stimulation, the kurtosis of the VHCF was larger in the second part of the tracks (10-20 s) compared with the first part $(0-10 \mathrm{~s})$ for all time lags (Fig. $4 G)$. In contrast, when the stimulus was present, the reverse was observed, i.e., the kurtosis of the VHCF was strongly reduced during the stimulation period (Fig. $4 H$ ). This reduction in kurtosis corresponds to reduced deviation from Gaussianity during stimulation compared with resting conditions. This result can be interpreted to indicate that stimulation leads to a reduction in the active component of vesicle motion upon stimulation, which is consistent with the overall reduction in vesicle displacement observed by both spatial analyses above (Figs. $4 B, E, F$ ) and with the predominant changes in the vesicle dynamical states we observed initially in vesicle subpopulations (Fig. 3). However, these changes in VHCF can also arise from rapid changes in the vesicle environment upon stimulation. Such changes could be caused, for example, by activation of a number of calcium-dependent signaling processes upon the increase in intracellular calcium during stimulation (see Discussion).

In summary, our spatial analyses of mobility over the entire bouton population suggest that vesicle motion undergoes activitydependent changes apparent as the reduction in overall vesicle displacement during stimulation. These changes are consistent with the
A
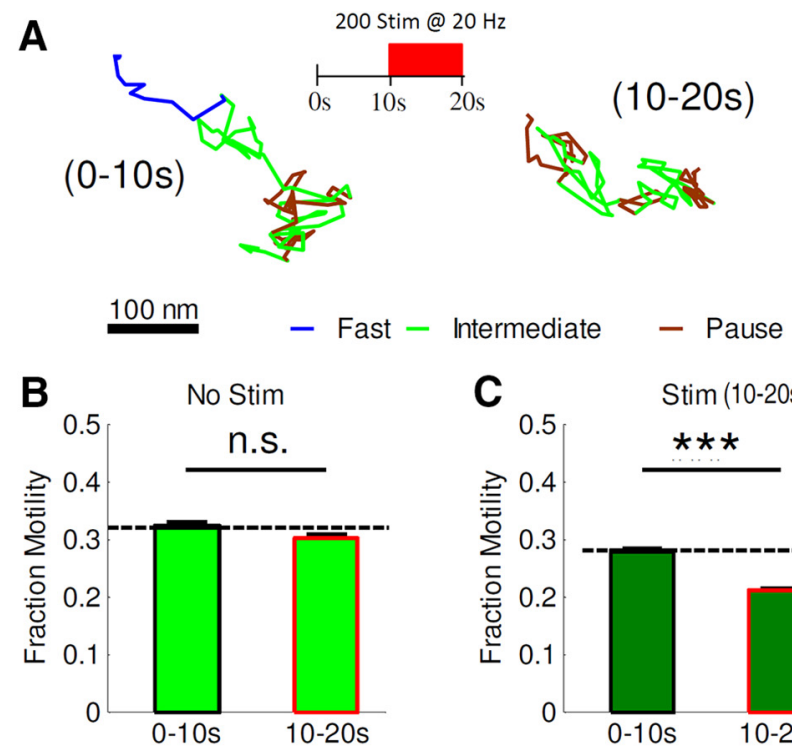

\section{D}
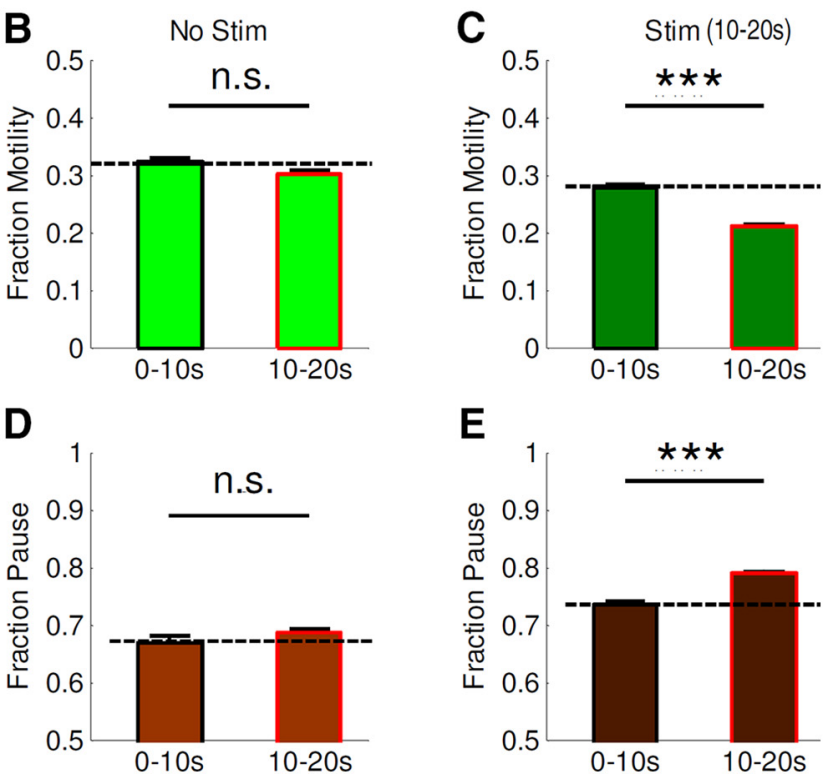

E

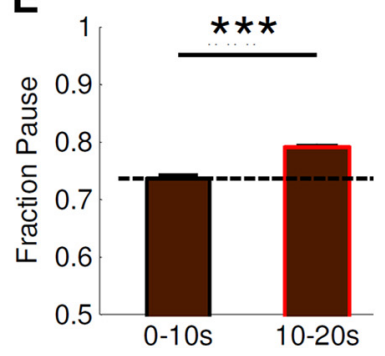

Figure 5. Reduction in time spent in fast correlated motion contributes to activity-evoked changes in vesicle mobility. $\boldsymbol{A}$, Sample vesicle track subdivided into three components of motion based on correlation analysis into periods of fast directed (blue), pausing (brown), and intermediate (Green) motion. Two parts of the sample track corresponding to vesicle motion during $0-10$ s and $10-20$ s periods are plotted separately for the presentation purposes only to highlight differences in vesicle motion at baseline and during stimulation. Scale bar, $100 \mathrm{~nm}$. $B, C$, Proportion of time vesicles spent in mobile states (fast and intermediate mobility) without $(\boldsymbol{B})$ and with $(\boldsymbol{C})$ stimulus applied during the $10-20$ s period. $n=26$ and 35 . D, E, Same as $\boldsymbol{B}$ and C for pausing. ${ }^{* * *} p<0.001$, two-tailed $t$ test in $\boldsymbol{B}-\boldsymbol{E}$. Error bars represent SEM.

reduction in directed motion upon stimulation, and/or calciumevoked changes in vesicle environment.

\section{Reduction in fast correlated motion contributes to activity-evoked changes in vesicle mobility}

To confirm the above results and determine further whether changes in directed motion contribute to reduction in vesicle mobility upon stimulation, we took advantage of a more restrictive motion analysis approach that combines both spatial and temporal characteristics of vesicle motion. This analysis smooths the raw track data, calculates the parameters of instantaneous velocity and directional change, and then determines a correlation of those parameters using a predefined threshold (see Materials and Methods; Gramlich and Klyachko, 2017).

We characterized three different components of vesicle trajectories based on the correlated directionality, velocity and time spent in a given motion as follows: fast directed mobility, pausing, and an intermediate mobility that encompasses all the remaining motion (Fig. 5A; Materials and Methods). Within these definitions, the fast directed mobility relates to the fastest component of directed motion; the pausing relates predominately to stalled motion and a slow component of diffusion; and intermediate mobility relates to the remaining motion, predominately 

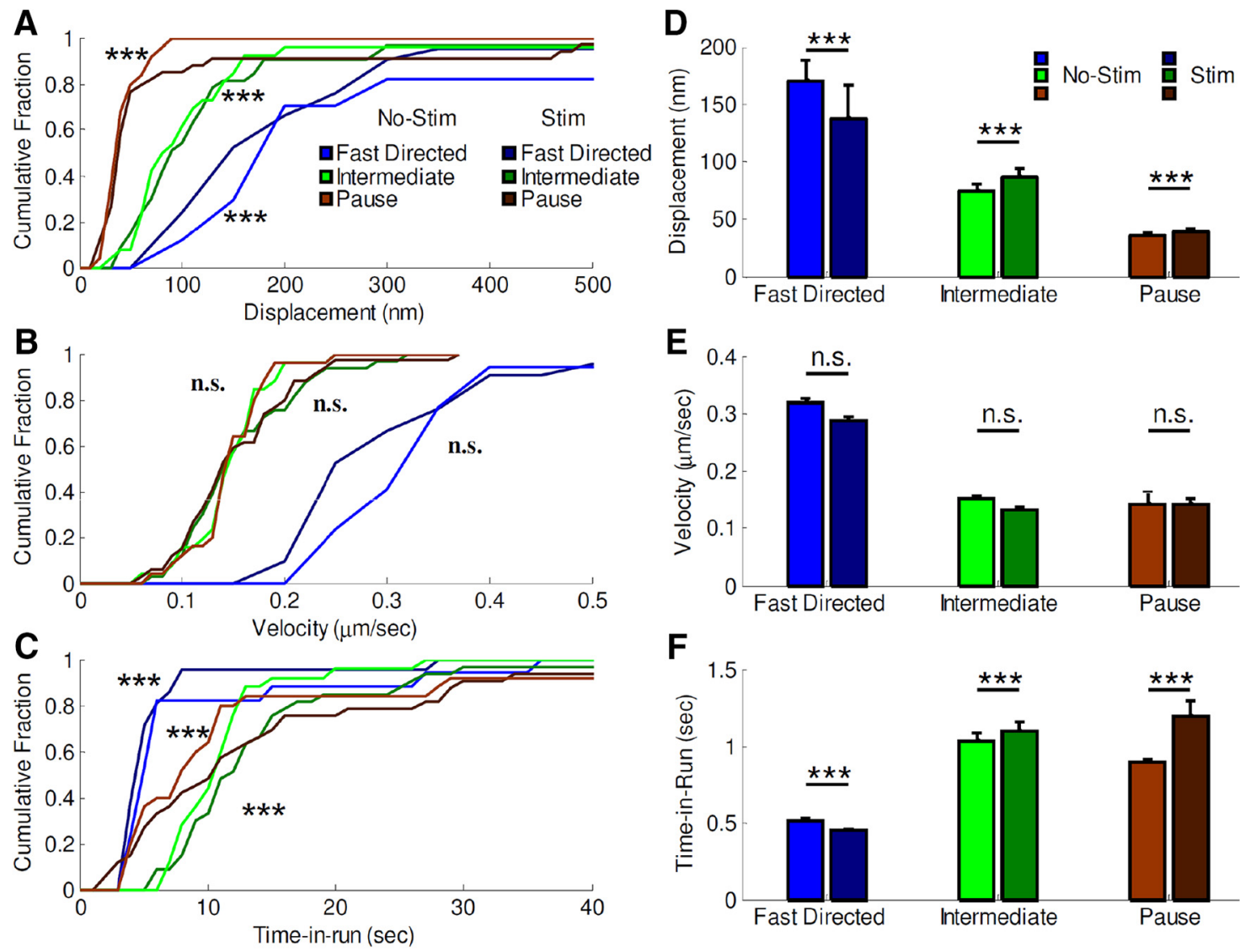

Figure 6. Reduction in motility mechanics of fast correlated motion evoked by activity. Cumulative $(\boldsymbol{A})$ and mean values $(\boldsymbol{D})$ of vesicle displacement without (light-colors) and with (dark-colors) stimulus for each motion component (fast directed, intermediate, pausing). Cumulative $(\boldsymbol{B})$ and mean values $(\boldsymbol{E})$ of average vesicle velocity during each motion component. Cumulative $(\boldsymbol{C})$ and mean values $(\boldsymbol{F})$ of time spent in each motion component. All metrics in $\boldsymbol{D}-\boldsymbol{E}$ are averaged for the entire observation period $(0-20 \mathrm{~s}) .{ }^{* * *} p<0.001$, two-tailed KS test. Error bars represent SEM.

faster component of diffusive motion and slower directed mobility. We used this analysis to specifically analyze the subpopulation of vesicles that exhibit at least one intermediate or fast directed run within the first $20 \mathrm{~s}$ of observation.

We first determined broadly whether this correlation analysis approach is sufficiently sensitive to permit detection of activityevoked changes in vesicle mobility across the entire vesicle population. In this analysis, we first performed a three-frame moving averaging of the raw data to reduce the component of noise in vesicle localization associated with limited localization precision (Peng et al., 2012), followed by correlation analysis on the smoothed data (see Materials and Methods). We then quantified the "mobile" fraction of vesicles (undergoing fast directed and intermediate mobility) compared with the paused vesicle fraction, before (0-10 s) and during (10-20 s) high-frequency stimulation (200 stimuli at $20 \mathrm{~Hz}$ ). In the absence of stimulation for the entire observation period, the fraction of mobile vesicles remained constant over time (Fig. $5 B ; p=0.81, t$ test; degrees of freedom $=198)$. In contrast, the mobile vesicle fraction decreased significantly in the presence of activity (10-20 s) compared with previous period ( $0-10 \mathrm{~s}$ ) of no activity (Fig. $5 C ; p=$ $4.9 \mathrm{E}-22$; degrees of freedom $=198$ ). Complementary to these changes, the paused fraction increased in the presence of activity (Fig. $5 E ; p=2.82 \mathrm{E}-13$; degrees of freedom $=198$ ), whereas it remained constant in the absence of stimulation (Fig. $5 D ; p=$ 0.66 ; degrees of freedom $=198$ ). The same activity-evoked changes in mobile and paused fractions were also observed when we compared across the two vesicle populations that did or did not have stimulation applied during $10-20$ s period, i.e., a significant reduction in mobile fraction between No Stim: $10-20 \mathrm{~s}$ and Stim: $10-20 \mathrm{~s}(p=3.3 \mathrm{E}-47$; degrees of freedom $=198)$, and a significant increase in paused fraction $(p=2.2 \mathrm{E}-38$; degrees of freedom $=198)$. We note that the fractional changes observed with this analysis $(\sim 10-25 \%)$ are somewhat larger but comparable to what could be expected from visually apparent changes in mobility averaged across the entire bouton population (Fig. 3). These results suggest that overall vesicle mobility decreases in the presence of stimulation, whereas pausing concomitantly increases. This result provides further support to our findings using other approaches that reduction in vesicle displacement is the predominant activity-evoked change in vesicle motion.

This analysis approach also allows us to quantify specific metrics of individual motion components including length of displacement, velocity, and time spent in each motion, thus allowing us to distinguish the mechanics of how each type of mobility was affected by stimulation (Fig. $6 A-F$ ). In this analysis we calculated each metric for the entire duration of observation (20 s) either at resting conditions when stimulation was absent entirely, or when stimulation was applied during $10-20 \mathrm{~s}$ periods of observation. The necessity of using the entire 20 s period of vesicle trajectory in this analysis was driven by the fact that due to stringency in identification of fast correlated runs in our algorithm, only a small subset of vesicles exhibited a fast run within the first $20 \mathrm{~s}$, and typically only a single run was observed within this time period. Thus, separate analyses of $0-10 \mathrm{~s}$ and $10-20 \mathrm{~s}$ periods was not feasible due to a small number of analyzable tracks. We thus 
compared the entire $0-20 \mathrm{~s}$ duration to increase the number of analyzable tracks and obtain statistically valid results. We found that activity affected the distance traveled in all three types of motion (Fig. $6 A, D$ ): the length of travel in fast correlated motion was reduced by $\sim 20 \%$ in the presence of activity $(p=1.85 \mathrm{E}-5$, KS test), whereas both intermediate mobility and pausing showed a corresponding increase in displacement by $\sim 15 \%$ and $\sim 10 \%$, respectively $(p=5.29 \mathrm{E}-74$ for intermediate, $p=1.85 \mathrm{E}-19$ for pause, KS test). We further found that the time spent in fast correlated motion decreased, whereas time spent in intermediate motion and pausing corresponding increased when stimulation was present (Fig. $6 C, F ; p=1.89 \mathrm{E}-15$ for all, KS test). Finally, we observed no significant changes in average velocity in any of the motion components (Fig. $6 B, E$; Fast: $p=0.139$; Intermediate: $p=0.143$; Pause: $p=0.965$ ) or the number of runs (Fast: $p=$ 0.3129; Intermediate: $p=0.986$; Pause: $p=0.216$, KS test; data not shown), although we note that velocities for intermediate mobility and pausing are at the lower limit of our experimental resolution, and thus exhibit artificially similar distributions. These results indicate that changes in vesicle displacement with activity arise from changes in the time spent in each type of motion, rather than from changes in the kinetics of individual motion components.

These results confirm and extend the above findings that vesicle mobility is reduced during neural activity, and this reduction is associated predominately with a decrease in the time spent in fast correlated/directed motion and concomitant increase in the time spent pausing.

\section{Discussion}

Using nanoscale resolution tracking of individual synaptic vesicles in hippocampal synapses and a set of complementary motion analysis tools we demonstrate that recently endocytosed vesicles undergo complex sets of dynamical states that include epochs of directed, diffusive and stalled motion. Most importantly, these analyses revealed that vesicle motion is modulated in an activitydependent manner apparent as the reduction in overall vesicle mobility in response to stimulation. These activity-dependent changes are consistent with the reduction in fast correlated (directed) vesicle motion and increased pausing upon stimulation. Our analyses further suggest that the effects of activity arise from changes in the time spent in each type of motion, rather than from changes in the kinetics of individual motion components. These results define the vesicle dynamical states during recycling and reveal their activity-dependent modulation, which may represent an important mechanism controlling vesicle availability for release during neuronal activity.

Current understanding of the spatial organization of synaptic vesicle pools suggests that vesicle lifecycle involves multiple translocations between the plasma membrane and the interior of the synaptic bouton. Upon endocytosis, vesicles transition toward the interior, where they are thought to be tethered to the actin cytoskeleton (Sudhof, 2004); subsequently they move back toward the plasma membrane, where vesicles join the readilyreleasable pool (RRP) and fuse upon stimulation. Our findings that vesicles undergo multiple bouts of directed, diffusive and stalled motion support the view that recently endocytosed vesicles are highly mobile inside the synaptic boutons (Kamin et al., 2010; Lee et al., 2012; Park et al., 2012) and undergo multiple transitions between several dynamical states. Discerning how the dynamical states we observed relate to specific transitions between synaptic vesicle pools will require future investigation because of the current difficulty of correlating the single-vesicle trajectory with the structural geometry of the synaptic terminal. Because high-resolution 3D structural analyses are time-intensive and could only be performed after single-vesicle imaging, correlating the vesicle track with the subsequently defined synaptic ultrastructure is complicated by a number of factors, including slow random synapse displacement over time (Lemke and Klingauf, 2005). Future studies may be able to use multicolor approaches to perform such colocalization measurements simultaneously. For example, the vesicle trajectory can be related to the locations of the reserve pool and the RRP using fluorescence tagging of known presynaptic tethering proteins, such as synapsins (Orenbuch et al., 2012; Shulman et al., 2015) and complexins (Yang et al., 2013; Wragg et al., 2015), respectively. However, we were limited in performing such multicolor colocalization experiments because even the slight spectral overlap of the synaptic structure tags with the single-vesicle label, which is unavoidable in such multicolor experiments, significantly reduces precision of vesicle localization in our experimental setup. Nevertheless, these findings provide a first step toward defining the dynamical correlates of different steps in the vesicle cycle.

The key advantage of our motion analyses tools is in the ability to detect activity-evoked changes in vesicle motion not previously seen in studies of synaptic vesicle dynamics (Lemke and Klingauf, 2005; Westphal et al., 2008). Indeed, findings that induction of LTP or LTD induces changes in the vesicle diffusion coefficient and spatial extent of vesicle motion (Lee et al., 2012) suggested that vesicle motion is modulated in an activity-dependent manner. Yet the basic diffusion analyses used previously did not detect measurable activity-evoked changes in vesicle dynamics (Lemke and Klingauf, 2005; Westphal et al., 2008). In contrast, our analyses of single-vesicle trajectories revealed that vesicle motion is indeed modulated by activity. Activity-evoked changes in vesicle motion were heterogeneous and apparent only in the subpopulation of boutons as either acceleration or deceleration in response to activity and corresponding changes of directed motion. Such heterogeneous and opposing changes are likely to partially mask each other in analyses of the entre vesicle population, which may explain why these effects of activity have not been previously detected. Because only a single vesicle is labeled in each bouton, our analyses do not distinguish whether the observed heterogeneity arise from different vesicle subpopulations within individual boutons or from heterogeneity of boutons themselves, both of which have been observed previously (Dobrunz and Stevens, 1997; Scanziani et al., 1998; Westphal et al., 2008; Kamin et al., 2010; Lee et al., 2012; Park et al., 2012). Also most previous analyses of vesicle mobility were performed at room temperature, a condition that could obscure the activity-dependent changes in vesicle dynamics. Indeed, lowering temperature reduces the baseline vesicle mobility (Lemke and Klingauf, 2005; Gaffield and Betz, 2007), and obscures the effects of stimulation on already strongly reduced mobility (Fig. $4 B$ ). The effects of lowering the temperature on vesicle mobility are also indirectly evident in the marked reduction in vesicle resupply at room temperatures (Pyott and Rosenmund, 2002; Volgushev et al., 2004; Micheva and Smith, 2005; Klyachko and Stevens, 2006). Moreover, recovery from synaptic depression is greatly accelerated by increasing temperature from $23^{\circ} \mathrm{C}$ to $37^{\circ} \mathrm{C}$ at the calyx of Held and hippocampal synapses (Klyachko and Stevens, 2006; Kushmerick et al., 2006), which has been proposed to be caused by a temperature-dependent acceleration of vesicle mobilization (Kushmerick et al., 2006).

The three analyses we used, each based on a different set of mathematical tools, provided strong support for the activity- 
dependent changes in vesicle motion at $37^{\circ} \mathrm{C}$. When applied to the entire vesicle population, we found that this activity-dependent modulation manifests as a reduction in overall vesicle mobility during stimulation. Specifically, using VHCF analysis we found a clear deviation of vesicle displacements from Gaussian (pure diffusive) at basal conditions, and observed that this deviation from diffusive motion is reduced during stimulation. Deviation from Gaussian distribution in VHCF analysis can be attributed either to periods of directed motion or to diffusion in a highly heterogeneous environment. Activity-dependent changes we observed may thus correspond to reduction in directed motion or due to changes in the vesicle environment upon stimulation.

The first possibility is consistent with the suggested roles of actin cytoskeleton and myosin motors in regulating vesicle recycling and translocation (Shupliakov et al., 2002; Peng et al., 2012; Messa et al., 2014; Wu et al., 2016). Recent studies suggested that refilling of release sites in cerebellar synapses depends on actin and myosin II (Miki et al., 2016), which is consistent with the earlier findings on the role of actin, myosin light chain kinase, and myosin II in the RRP refilling in the calyx of Held (Srinivasan et al., 2008; Lee et al., 2010), brainstem synapses (GonzálezForero et al., 2012), and hippocampal synapses (Peng et al., 2012; Chandrasekar et al., 2013). Notably, Myosin II possesses a limited processive ability (Neco et al., 2004; Norstrom et al., 2010), and is thought to function predominately to generate tension and promote actin dynamics required for processive motion of other myosin isoforms (Semenova et al., 2008). Indeed, in addition to myosin II, myosin-Va has been detected in presynaptic boutons and identified as a synaptic vesicle associated protein (Ohyama et al., 2001; Takamori et al., 2006). Moreover, our single-vesicle studies revealed a direct role for myosin- $\mathrm{V}$ in supporting directed vesicle motion both within and between hippocampal boutons (Gramlich and Klyachko, 2017). Although the molecular identity of all the components supporting directed vesicle motion inside synaptic boutons awaits further investigation, it is noteworthy that myosin- $\mathrm{V}$ is known to undergo a transition from a processive motor to a tether in a calcium-dependent manner (Krementsov et al., 2004), which is consistent with the effects of activity on vesicle mobility. Activity-evoked reduction in directed motion is also consistent with our correlation analysis of individual motion metrics, indicating that the time vesicles spent in fast correlated motion is reduced during stimulation.

Changes in the vesicle environment may also be a contributing factor that mediates effects of activity on vesicle motion, given the large number of calcium-dependent proteins at the synaptic boutons (Neher and Sakaba, 2008). This later possibility can arise for example from relocation or transformational changes of signaling molecules upon elevation in intracellular calcium induced by stimulation, thereby leading to changes in vesicle environment and altering vesicle mobility. Our observation that overall vesicle mobility decreases with activity may also relate to an alternative and/or parallel pathway of vesicle-endosomal fusion. Synaptic vesicles have been observed to fuse with large endosomes during their life-cycle (Heuser and Reese, 1973; Kokotos and Cousin, 2015), although prevalence of this process during vesicle recycling in central synapses remains debatable (Murthy and Stevens, 1998; Lee et al., 2012; Kokotos and Cousin, 2015). The reduction in synaptic vesicle mobility may thus in part be due to capture by larger endosomes that likely have a slower overall mobility. Depending on kinetics of this process, such a mechanism may be initially reflected in vesicle pausing, but eventually will lead to vesicle disappearance due to dispersion of the dye and loss of vesicle detection. Future studies correlating patterns of vesicle behavior and disappearance will help better understand the prevalence and function of this pathway. We also note that prolonged high-frequency stimulation was found to cause an increase in bouton volume (Chéreau et al., 2017), which can be expected to reduce molecular crowding and increase vesicle mobility, in contrast with our observations. However, the reported increase in bouton size occurred after several minutes of stimulation, whereas 10-s-long stimulation used in our study is expected to cause at most a few percentage changes in bouton size. Such small changes are likely within the uncertainty of our algorithms and would not be expected to produce measurable effects on vesicle mobility in our measurements.

In summary, our results suggest that, upon endocytosis, vesicles undergo a complex and activity-regulated translocation process to reach their destinations within the synaptic boutons. Future studies will be needed to reveal the complex molecular mechanisms by which activity regulates vesicle dynamics. Nevertheless, activity-dependent modulation of vesicle mobility may represent an important mechanism controlling vesicle availability and neurotransmitter release during heightened neural activity.

\section{References}

Ahmed WW, Saif TA (2014) Active transport of vesicles in neurons is modulated by mechanical tension. Sci Rep 4:4481. CrossRef Medline

Bal M, Leitz J, Reese AL, Ramirez DM, Durakoglugil M, Herz J, Monteggia LM, Kavalali ET (2013) Reelin mobilizes a VAMP7-dependent synaptic vesicle pool and selectively augments spontaneous neurotransmission. Neuron 80:934-946. CrossRef Medline

Chandrasekar I, Huettner JE, Turney SG, Bridgman PC (2013) Myosin II regulates activity dependent compensatory endocytosis at central synapses. J Neurosci 33:16131-16145. CrossRef Medline

Chéreau R, Saraceno GE, Angibaud J, Cattaert D, Nägerl UV (2017) Superresolution imaging reveals activity-dependent plasticity of axon morphology linked to changes in action potential conduction velocity. Proc Natl Acad Sci U S A 114:1401-1406. CrossRef Medline

Chi P, Greengard P, Ryan TA (2003) Synaptic vesicle mobilization is regulated by distinct synapsin I phosphorylation pathways at different frequencies. Neuron 38:69-78. CrossRef Medline

Chung C, Barylko B, Leitz J, Liu X, Kavalali ET (2010) Acute dynamin inhibition dissects synaptic vesicle recycling pathways that drive spontaneous and evoked neurotransmission. J Neurosci 30:1363-1376. CrossRef Medline

Darcy KJ, Staras K, Collinson LM, Goda Y (2006) Constitutive sharing of recycling synaptic vesicles between presynaptic boutons. Nat Neurosci 9:315-321. CrossRef Medline

Del Pópolo MG, Voth G (2004) On the structure and dynamics of ionic liquids. J Phys Chem B 108:1744-1752. CrossRef

Dobrunz LE, Stevens CF (1997) Heterogeneity of release probability, facilitation, and depletion at central synapses. Neuron 18:995-1008. CrossRef Medline

Fernández-Alfonso T, Ryan TA (2006) The efficiency of the synaptic vesicle cycle at central nervous system synapses. Trends Cell Biol 16:413-420. CrossRef Medline

Fredj NB, Burrone J (2009) A resting pool of vesicles is responsible for spontaneous vesicle fusion at the synapse. Nat Neurosci 12:751-758. CrossRef Medline

Gaffield MA, Betz WJ (2007) Synaptic vesicle mobility in mouse motor nerve terminals with and without synapsin. J Neurosci 27:13691-13700. CrossRef Medline

Gaffield MA, Rizzoli SO, Betz WJ (2006) Mobility of synaptic vesicles in different pools in resting and stimulated frog motor nerve terminals. Neuron 51:317-325. CrossRef Medline

González-Forero D, Montero F, García-Morales V, Domínguez G, GómezPérez L, García-Verdugo JM, Moreno-López B (2012) Endogenous Rho-kinase signaling maintains synaptic strength by stabilizing the size of the readily releasable pool of synaptic vesicles. J Neurosci 32:68-84. CrossRef Medline

Gramlich MW, Klyachko VA (2017) Actin/myosin-V- and activity-dependent inter-synaptic vesicle exchange in central neurons. Cell Rep 18: 2096-2104. CrossRef Medline 
Harata N, Pyle JL, Aravanis AM, Mozhayeva M, Kavalali ET, Tsien RW (2001) Limited numbers of recycling vesicles in small CNS nerve terminals: implications for neural signaling and vesicular cycling. Trends Neurosci 24:637-643. CrossRef Medline

Henkel AW, Simpson LL, Ridge RM, Betz WJ (1996) Synaptic vesicle movements monitored by fluorescence recovery after photobleaching in nerve terminals stained with FM1-43. J Neurosci 16:3960-3967. Medline

Heuser JE, Reese TS (1973) Evidence for recycling of synaptic vesicle membrane during transmitter release at the frog neuromuscular junction. J Cell Biol 57:315-344. CrossRef Medline

Hua Z, Leal-Ortiz S, Foss SM, Waites CL, Garner CC, Voglmaier SM, Edwards RH (2011) v-SNARE composition distinguishes synaptic vesicle pools. Neuron 71:474-487. CrossRef Medline

Huet S, Karatekin E, Tran VS, Fanget I, Cribier S, Henry JP (2006) Analysis of transient behavior in complex trajectories: application to secretory vesicle dynamics. Biophys J 91:3542-3559. CrossRef Medline

Jaqaman K, Loerke D, Mettlen M, Kuwata H, Grinstein S, Schmid SL, Danuser G (2008) Robust single-particle tracking in live-cell time-lapse sequences. Nat Methods 5:695-702. CrossRef Medline

Joensuu M, Padmanabhan P, Durisic N, Bademosi AT, Cooper-Williams E, Morrow IC, Harper CB, Jung W, Parton RG, Goodhill GJ, Papadopulos A, Meunier FA (2016) Subdiffractional tracking of internalized molecules reveals heterogeneous motion states of synaptic vesicles. J Cell Biol 215:277-292. CrossRef Medline

Kamin D, Lauterbach MA, Westphal V, Keller J, Schönle A, Hell SW, Rizzoli SO (2010) High- and low-mobility stages in the synaptic vesicle cycle. Biophys J 99:675-684. CrossRef Medline

Klyachko VA, Stevens CF (2006) Temperature-dependent shift of balance among the components of short-term plasticity in hippocampal synapses. J Neurosci 26:6945-6957. CrossRef Medline

Kokotos AC, Cousin MA (2015) Synaptic vesicle generation from central nerve terminal endosomes. Traffic 16:229-240. CrossRef Medline

Kraszewski K, Daniell L, Mundigl O, de Camilli P (1996) Mobility of synaptic vesicles in nerve endings monitored by recovery from photobleaching of synaptic vesicle-associated fluorescence. J Neurosci 16:5905-5913. Medline

Krementsov DN, Krementsova EB, Trybus KM (2004) Myosin V: regulation by calcium, calmodulin, and the tail domain. J Cell Biol 164:877-886. CrossRef Medline

Kushmerick C, Renden R, von Gersdorff H (2006) Physiological temperatures reduce the rate of vesicle pool depletion and short-term depression via an acceleration of vesicle recruitment. J Neurosci 26:1366-1377. CrossRef Medline

Lee JS, Ho WK, Lee SH (2010) Post-tetanic increase in the fast-releasing synaptic vesicle pool at the expense of the slowly releasing pool. J Gen Physiol 136:259-272. CrossRef Medline

Lee S, Jung KJ, Jung HS, Chang S (2012) Dynamics of multiple trafficking behaviors of individual synaptic vesicles revealed by quantum-dot based presynaptic probe. PLoS One 7:e38045. CrossRef Medline

Leitz J, Kavalali ET (2011) $\mathrm{Ca}^{2+}$ influx slows single synaptic vesicle endocytosis. J Neurosci 31:16318-16326. CrossRef Medline

Lemke EA, Klingauf J (2005) Single synaptic vesicle tracking in individual hippocampal boutons at rest and during synaptic activity. J Neurosci 25:11034-11044. CrossRef Medline

Manzo C, Garcia-Parajo MF (2015) A review of progress in single particle tracking: from methods to biophysical insights. Rep Prog Phys 78:124601. CrossRef Medline

Maschi D, Klyachko VA (2015) A nanoscale resolution view on synaptic vesicle dynamics. Synapse 69:256-267. CrossRef Medline

Messa M, Fernández-Busnadiego R, Sun EW, Chen H, Czapla H, Wrasman K, Wu Y, Ko G, Ross T, Wendland B, De Camilli P (2014) Epsin deficiency impairs endocytosis by stalling the actin-dependent invagination of endocytic clathrin-coated pits. eLife 3:e03311. CrossRef Medline

Micheva KD, Smith SJ (2005) Strong effects of subphysiological temperature on the function and plasticity of mammalian presynaptic terminals. J Neurosci 25:7481-7488. CrossRef Medline

Miki T, Malagon G, Pulido C, Llano I, Neher E, Marty A (2016) Actin- and myosin-dependent vesicle loading of presynaptic docking sites prior to exocytosis. Neuron 91:808-823. CrossRef Medline

Monnier N, Guo SM, Mori M, He J, Lénárt P, Bathe M (2012) Bayesian approach to MSD-based analysis of particle motion in live cells. Biophys J 103:616-626. CrossRef Medline
Murthy VN, Stevens CF (1998) Synaptic vesicles retain their identity through the endocytic cycle. Nature 392:497-501. CrossRef Medline

Neco P, Giner D, Viniegra S, Borges R, Villarroel A, Gutiérrez LM (2004) New roles of myosin II during vesicle transport and fusion in chromaffin cells. J Biol Chem 279:27450-27457. CrossRef Medline

Neher E, Sakaba T (2008) Multiple roles of calcium ions in the regulation of neurotransmitter release. Neuron 59:861-872. CrossRef Medline

Norstrom MF, Smithback PA, Rock RS (2010) Unconventional processive mechanics of non-muscle myosin IIB. J Biol Chem 285:26326-26334. CrossRef Medline

Ohyama A, Komiya Y, Igarashi M (2001) Globular tail of myosin-V is bound to vamp/synaptobrevin. Biochem Biophys Res Commun 280: 988-991. CrossRef Medline

Orenbuch A, Shalev L, Marra V, Sinai I, Lavy Y, Kahn J, Burden JJ, Staras K, Gitler D (2012) Synapsin selectively controls the mobility of resting pool vesicles at hippocampal terminals. J Neurosci 32:3969-3980. CrossRef Medline

Park H, Li Y, Tsien RW (2012) Influence of synaptic vesicle position on release probability and exocytotic fusion mode. Science 335:1362-1366. CrossRef Medline

Peng A, Rotman Z, Deng PY, Klyachko VA (2012) Differential motion dynamics of synaptic vesicles undergoing spontaneous and activity-evoked endocytosis. Neuron 73:1108-1115. CrossRef Medline

Pyott SJ, Rosenmund C (2002) The effects of temperature on vesicular supply and release in autaptic cultures of rat and mouse hippocampal neurons. J Physiol 539:523-535. CrossRef Medline

Raingo J, Khvotchev M, Liu P, Darios F, Li YC, Ramirez DM, Adachi M, Lemieux P, Toth K, Davletov B, Kavalali ET (2012) VAMP4 directs synaptic vesicles to a pool that selectively maintains asynchronous neurotransmission. Nat Neurosci 15:738-745. CrossRef Medline

Sara Y, Virmani T, Deák F, Liu X, Kavalali ET (2005) An isolated pool of vesicles recycles at rest and drives spontaneous neurotransmission. Neuron 45:563-573. CrossRef Medline

Scanziani M, Gähwiler BH, Charpak S (1998) Target cell-specific modulation of transmitter release at terminals from a single axon. Proc Natl Acad Sci U S A 95:12004-12009. CrossRef Medline

Schikorski T (2014) Readily releasable vesicles recycle at the active zone of hippocampal synapses. Proc Natl Acad Sci U S A 111:5415-5420. CrossRef Medline

Schikorski T, Stevens CF (2001) Morphological correlates of functionally defined synaptic vesicle populations. Nat Neurosci 4:391-395. CrossRef Medline

Semenova I, Burakov A, Berardone N, Zaliapin I, Slepchenko B, Svitkina T, Kashina A, Rodionov V (2008) Actin dynamics is essential for myosinbased transport of membrane organelles. Curr Biol 18:1581-1586. CrossRef Medline

Shtrahman M, Yeung C, Nauen DW, Bi GQ, Wu XL (2005) Probing vesicle dynamics in single hippocampal synapses. Biophys J 89:3615-3627. CrossRef Medline

Shulman Y, Stavsky A, Fedorova T, Mikulincer D, Atias M, Radinsky I, Kahn J, Slutsky I, Gitler D (2015) ATP binding to synaspsin IIa regulates usage and clustering of vesicles in terminals of hippocampal neurons. J Neurosci 35:985-998. CrossRef Medline

Shupliakov O, Bloom O, Gustafsson JS, Kjaerulff O, Low P, Tomilin N, Pieribone VA, Greengard P, Brodin L (2002) Impaired recycling of synaptic vesicles after acute perturbation of the presynaptic actin cytoskeleton. Proc Natl Acad Sci U S A 99:14476-14481. CrossRef Medline

Skaug MJ, Mabry JN, Schwartz DK (2014) Single-molecule tracking of polymer surface diffusion. J Am Chem Soc 136:1327-1332. CrossRef Medline

Srinivasan G, Kim JH, von Gersdorff H (2008) The pool of fast releasing vesicles is augmented by myosin light chain kinase inhibition at the calyx of Held synapse. J Neurophysiol 99:1810-1824. CrossRef Medline

Staras K, Branco T, Burden JJ, Pozo K, Darcy K, Marra V, Ratnayaka A, Goda Y (2010) A vesicle superpool spans multiple presynaptic terminals in hippocampal neurons. Neuron 66:37-44. CrossRef Medline

Sudhof TC (2004) The synaptic vesicle cycle. Annu Rev Neurosci 27:509_ 547. CrossRef Medline

Takamori S, Holt M, Stenius K, Lemke EA, Gronborg M, Riedel D, Urlaub H, Schenck S, Brugger B, Ringler P, Müller SA, Rammner B, Gräter F, Hub JS, De Groot BL, Mieskes G, Moriyama Y, Klingauf J, Grubmüller H, Heuser J, et al. (2006) Molecular anatomy of a trafficking organelle. Cell 127:831-846. CrossRef Medline 
Volgushev M, Kudryashov I, Chistiakova M, Mukovski M, Niesmann J, Eysel UT (2004) Probability of transmitter release at neocortical synapses at different temperatures. J Neurophysiol 92:212-220. CrossRef Medline

Watanabe S, Liu Q, Davis MW, Hollopeter G, Thomas N, Jorgensen NB, Jorgensen EM (2013) Ultrafast endocytosis at Caenorhabditis elegans neuromuscular junctions. eLife 2:e00723. CrossRef Medline

Watanabe S, Lehmann M, Hujber E, Fetter RD, Richards J, Söhl-Kielczynski B, Felies A, Rosenmund C, Schmoranzer J, Jorgensen EM (2014) Nanometer-resolution fluorescence electron microscopy (nano-EM) in cultured cells. Methods Mol Biol 1117:503-526. CrossRef Medline

Westphal V, Rizzoli SO, Lauterbach MA, Kamin D, Jahn R, Hell SW (2008) Video-rate far-field optical nanoscopy dissects synaptic vesicle movement. Science 320:246-249. CrossRef Medline

Wragg RT, Gouzer G, Bai J, Arianna G, Ryan TA, Dittman JS (2015) Synap- tic activity regulates the abundance and binding of complexin. Biophys J 108:1318-1329. CrossRef Medline

Wu XS, Lee SH, Sheng J, Zhang Z, Zhao WD, Wang D, Jin Y, Charnay P, Ervasti JM, Wu LG (2016) Actin is crucial for all kinetically distinguishable forms of endocytosis at synapses. Neuron 92:1020-1035. CrossRef Medline

Wu Y, Yeh FL, Mao F, Chapman ER (2009) Biophysical characterization of styryl dye-membrane interactions. Biophys J 97:101-109. CrossRef Medline

Yang X, Cao P, Südhof TC (2013) Deconstructing complexin function in activating and clamping $\mathrm{Ca}^{2+}$-triggered exocytosis by comparing knockout and knockdown phenotypes. Proc Natl Acad Sci U S A 110:2077720782. CrossRef Medline

Yeung C, Shtrahman M, Wu XL (2007) Stick-and-diffuse and caged diffusion: a comparison of two models of synaptic vesicle dynamics. Biophys J 92:2271-2280. CrossRef Medline 